$\Psi$

UITS RT WORKSHOPS

Introduction to Information

Visualization 
Jefferson Davis, Research Analytics

\section{Statistical Graphics}




\section{Statistical Graphics}

\begin{tabular}{|c|c|}
\hline$x$ & $y=x^{2}$ \\
\hline 0.0 & 0.00 \\
\hline 0.1 & 0.01 \\
\hline 0.2 & 0.04 \\
\hline 0.3 & 0.09 \\
\hline 0.4 & 0.16 \\
\hline 0.5 & 0.25 \\
\hline 0.6 & 0.36 \\
\hline 0.7 & 0.49 \\
\hline 0.8 & 0.64 \\
\hline 0.9 & 0.81 \\
\hline 1.0 & 1.00 \\
\hline 1.1 & 1.21 \\
\hline 1.2 & 1.44 \\
\hline 1.3 & 1.69 \\
\hline
\end{tabular}

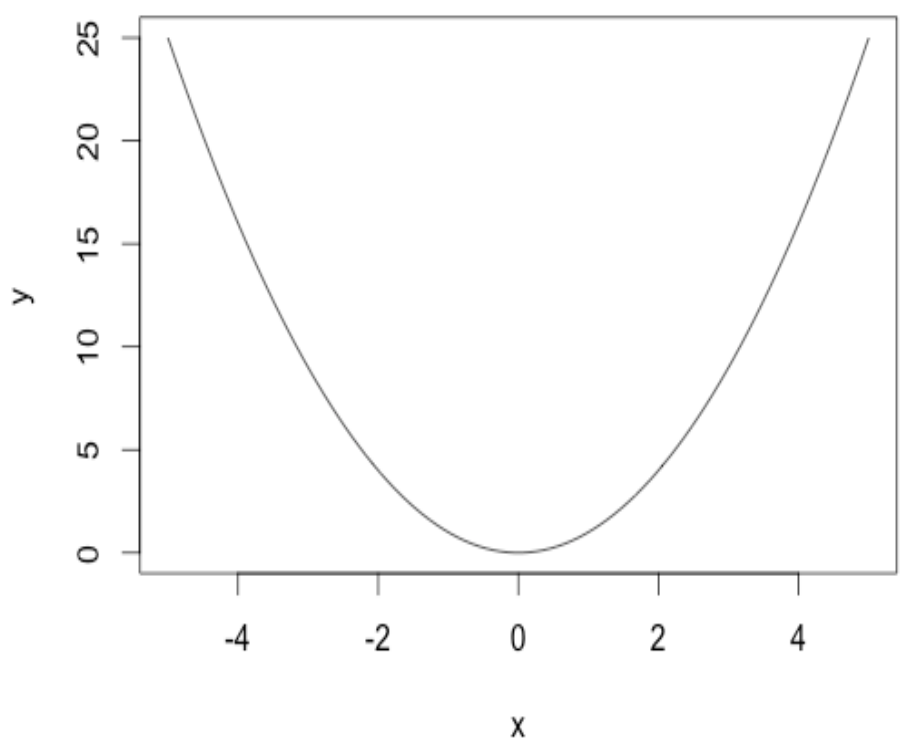




\section{Statistical Graphics}

Graphics can convey meaning without displaying any particular quantitative data. 


\section{Statistical Graphics}

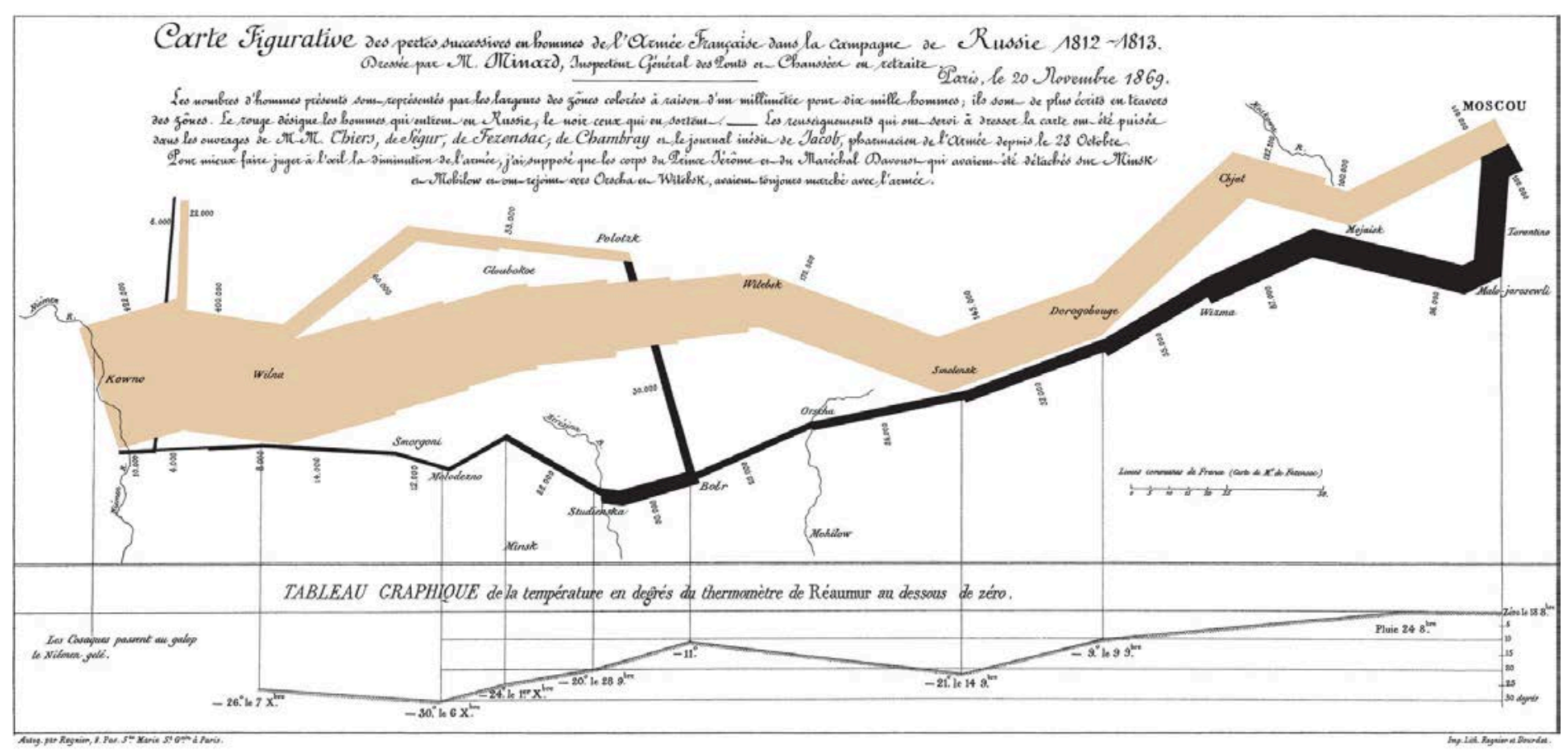




\section{Statistical Graphics}

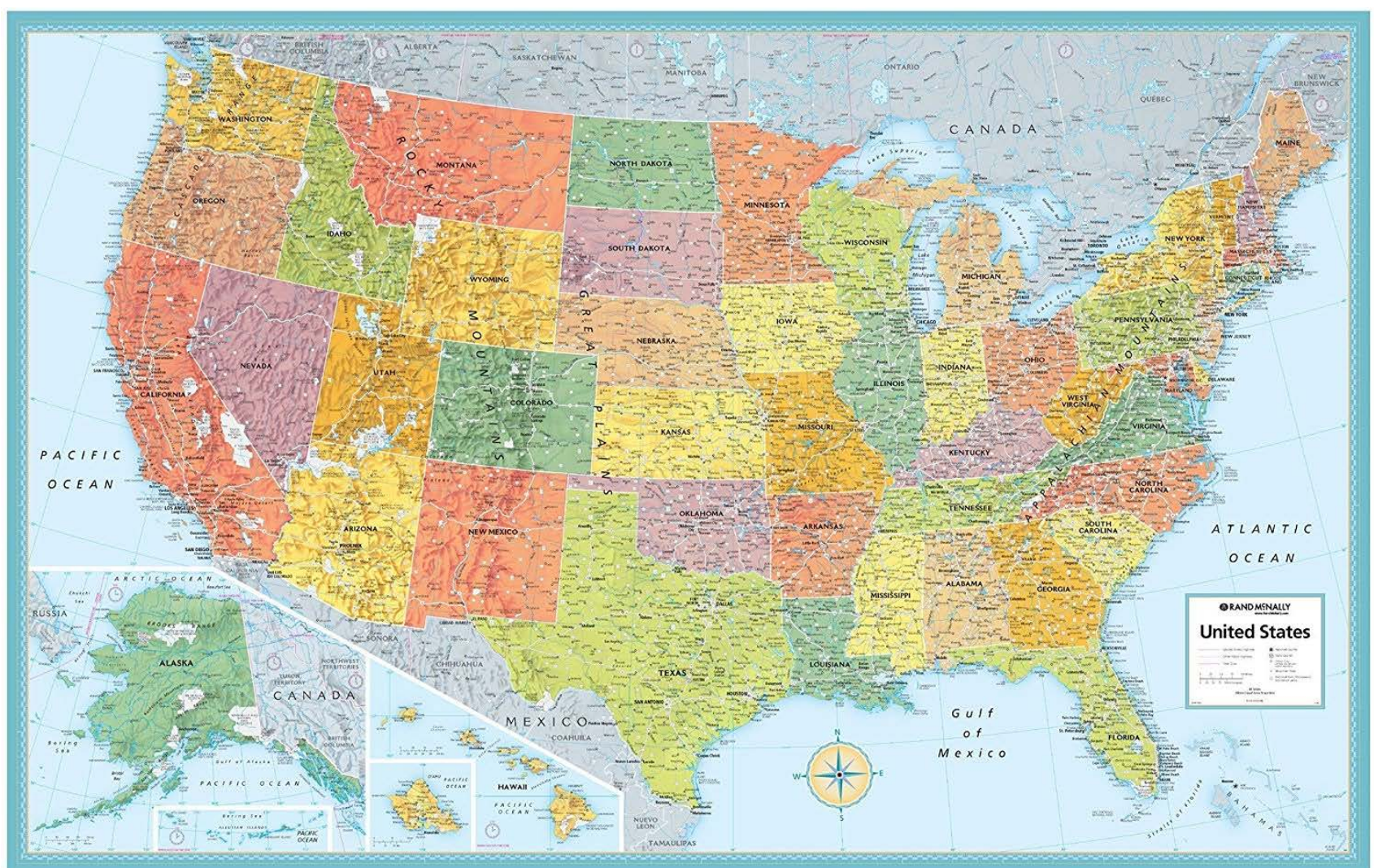




\section{The dataset}

The diamond dataset

\begin{tabular}{|llllllllll|} 
carat & cut & color & clarity & depth & table & price & x & y & z \\
& & & & & & & & & \\
0.23 & Ideal & E & SI2 & 61.5 & 55 & 326 & 3.95 & 3.98 & 2.43 \\
\hline & & & & & & & & & \\
0.21 & Premium E & SI1 & 59.8 & 61 & 326 & 3.89 & 3.84 & 2.31 \\
\hline & & & & & & & & & \\
0.23 & Good & E & VS1 & 56.9 & 65 & 327 & 4.05 & 4.07 & 2.31 \\
\hline & & & & & & & & & \\
0.29 & Premium I & VS2 & 62.4 & 58 & 334 & 4.2 & 4.23 & 2.63 \\
\hline & & & & & & & & & \\
0.31 & Good & J & SI2 & 63.3 & 58 & 335 & 4.34 & 4.35 & 2.75 \\
\hline & Very & & & & & & & & \\
0.24 & Good & J & VVS2 & 62.8 & 57 & 336 & 3.94 & 3.96 & 2.48 \\
\hline
\end{tabular}




\section{The dataset}
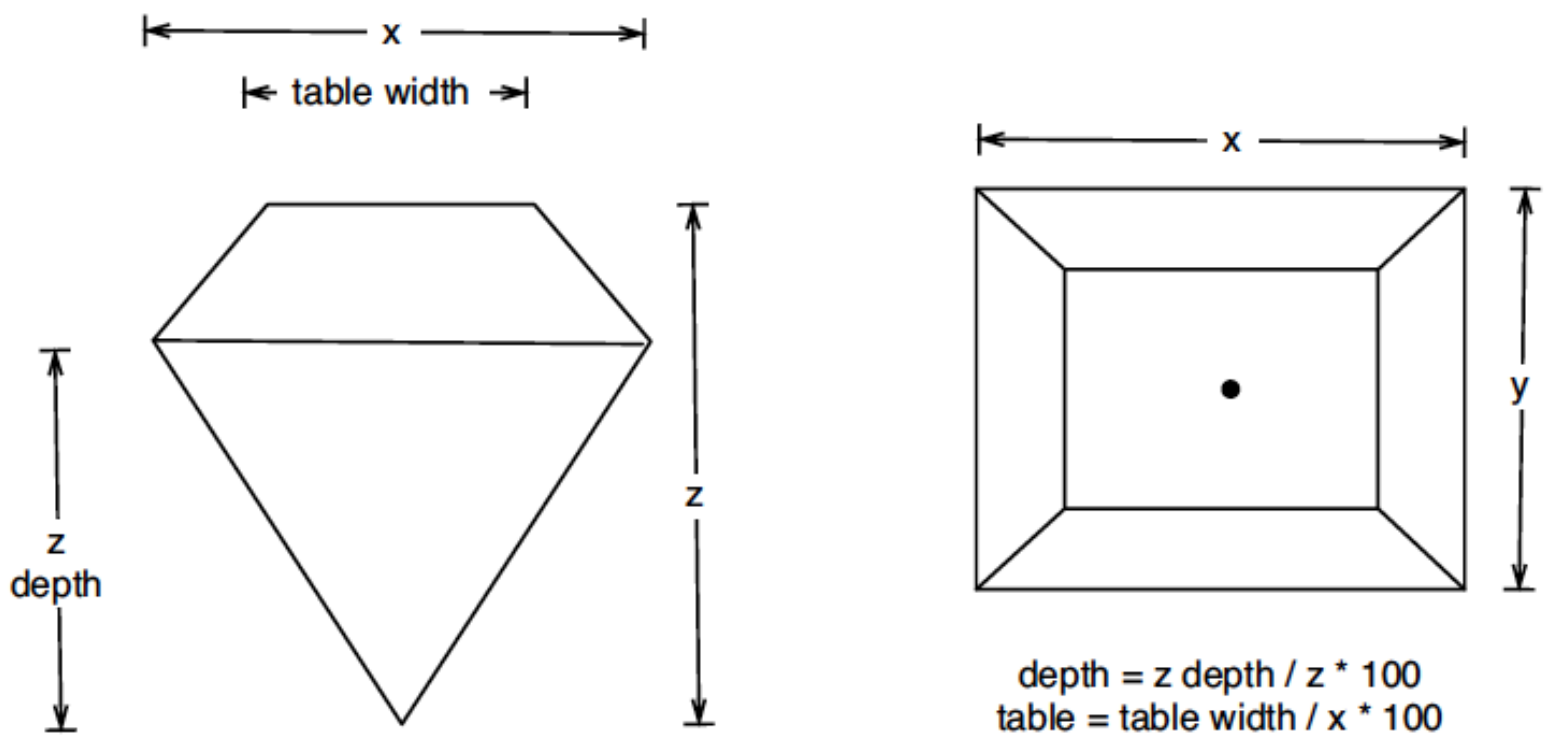

depth $=z$ depth $/ z^{*} 100$

table $=$ table width $/ x{ }^{*} 100$ 


\section{The dataset}

\begin{tabular}{|c|c|c|c|c|}
\hline \multicolumn{5}{|c|}{$\begin{array}{l}\text { library (ggplot2) } \\
\text { head(diamonds) [,1:4] }\end{array}$} \\
\hline & $\begin{array}{l}\text { A tibb } \\
\text { carat } \\
\langle\mathrm{dbl}\rangle\end{array}$ & le: $\begin{array}{r}6 \times 4 \\
\text { cut } \\
\langle\text { ord }>\end{array}$ & $\begin{array}{l}\text { color } \\
\text { <ord }\rangle\end{array}$ & $\begin{array}{r}\text { clarity } \\
\text { <ord }\end{array}$ \\
\hline & 0.23 & Ideal & E & SI2 \\
\hline & 0.21 & Premium & $\mathrm{E}$ & SI1 \\
\hline & 0.23 & Good & $\mathrm{E}$ & VS1 \\
\hline & 0.29 & Premium & I & VS2 \\
\hline & 0.31 & Good & J & $\mathrm{SI} 2$ \\
\hline & 0.24 & Very Good & J & VVS2 \\
\hline
\end{tabular}




\section{qplot()}

plot (diamonds\$carat, diamonds\$price)

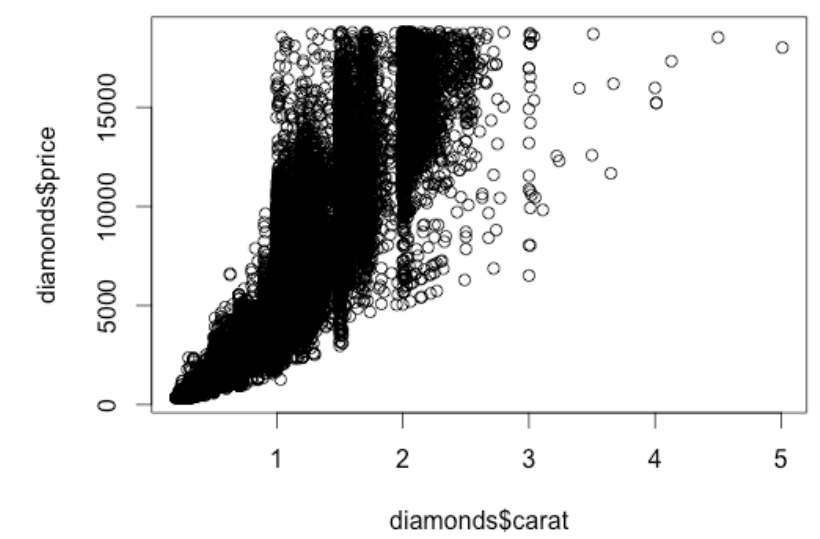

$$
\begin{aligned}
& \text { qplot (carat, price, } \\
& \text { data }=\text { diamonds) }
\end{aligned}
$$

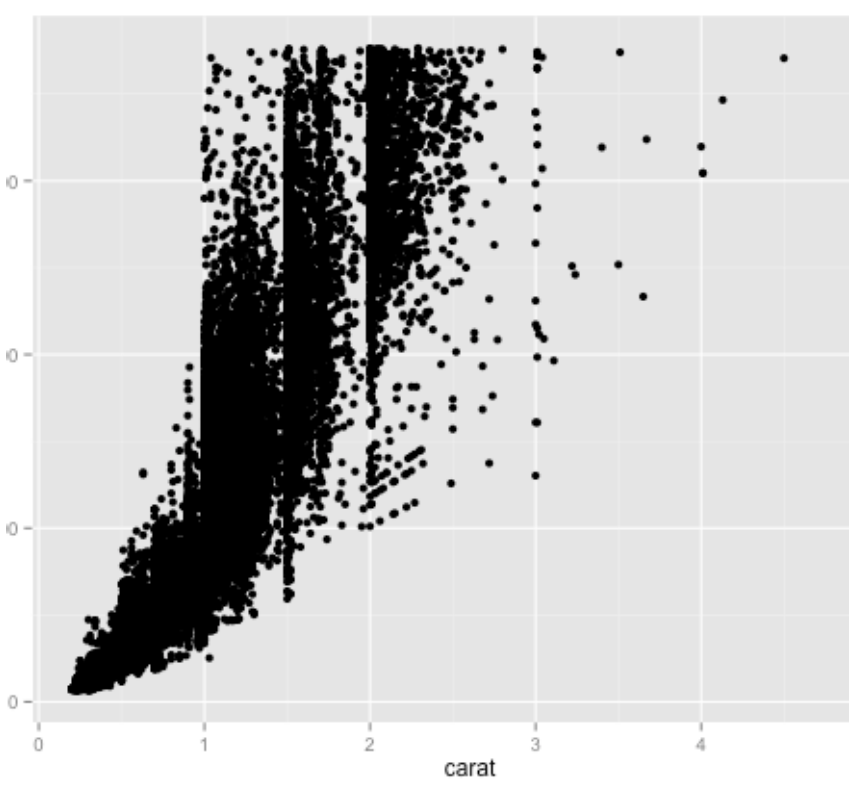




\section{qplot()}

qplot (diamonds\$carat, diamonds\$price))

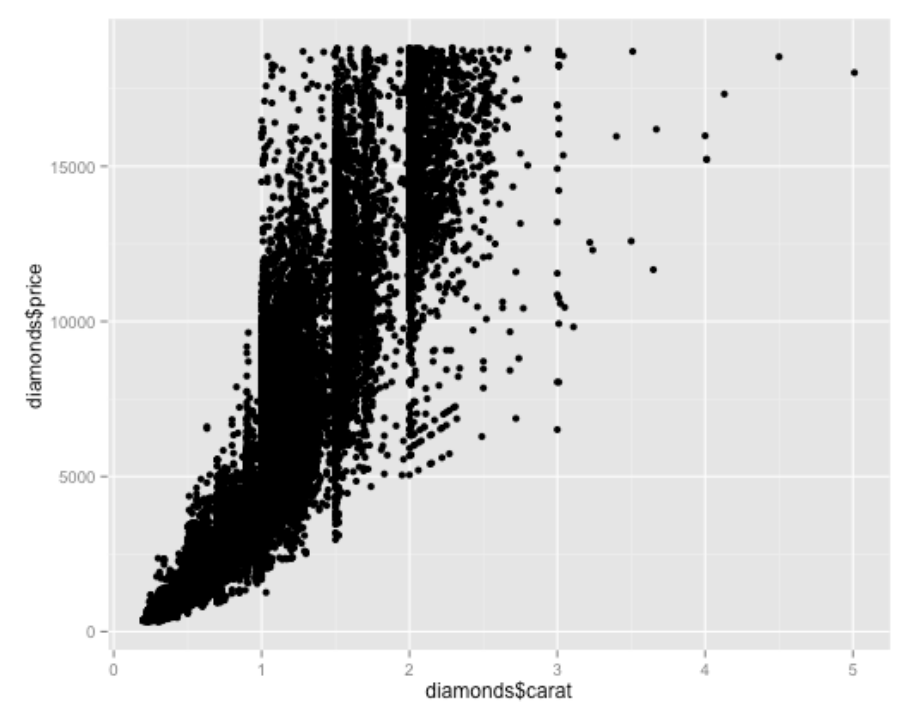




\section{qplot()}

If we add a title we will redraw

qplot (carat,

price,

data=diamonds ) +

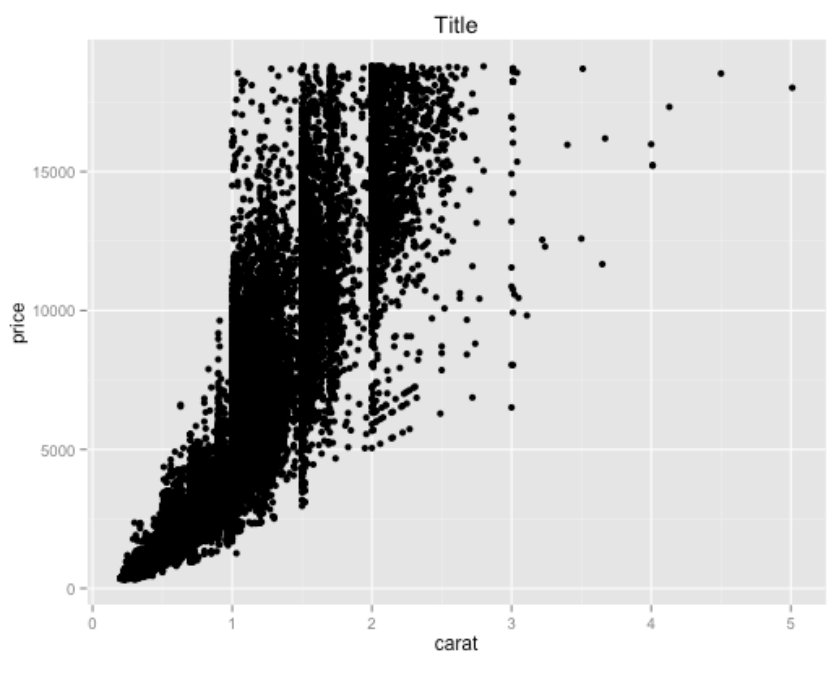

labs(title = "Title") 


\section{qplot()}

plot(diamonds\$carat) qplot(diamonds\$carat)
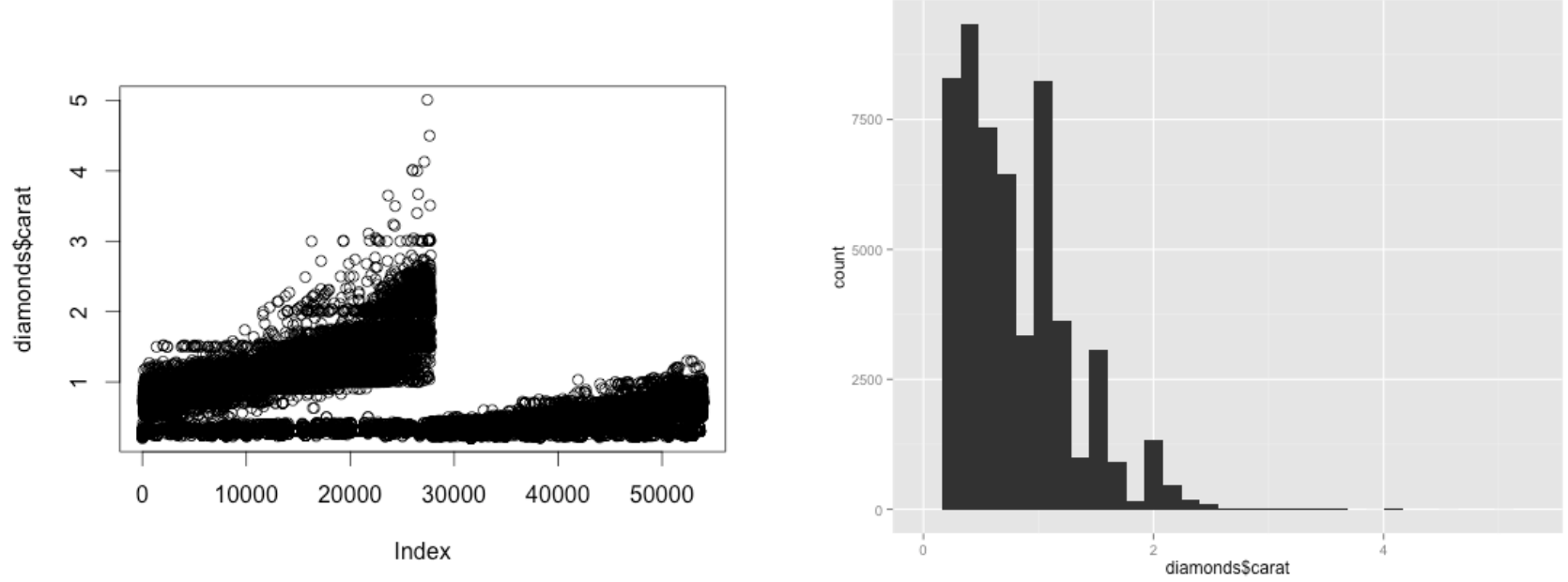


\section{qplot()}

qplot (carat, price, data = diamonds, shape = cut, color $=$ color $)$

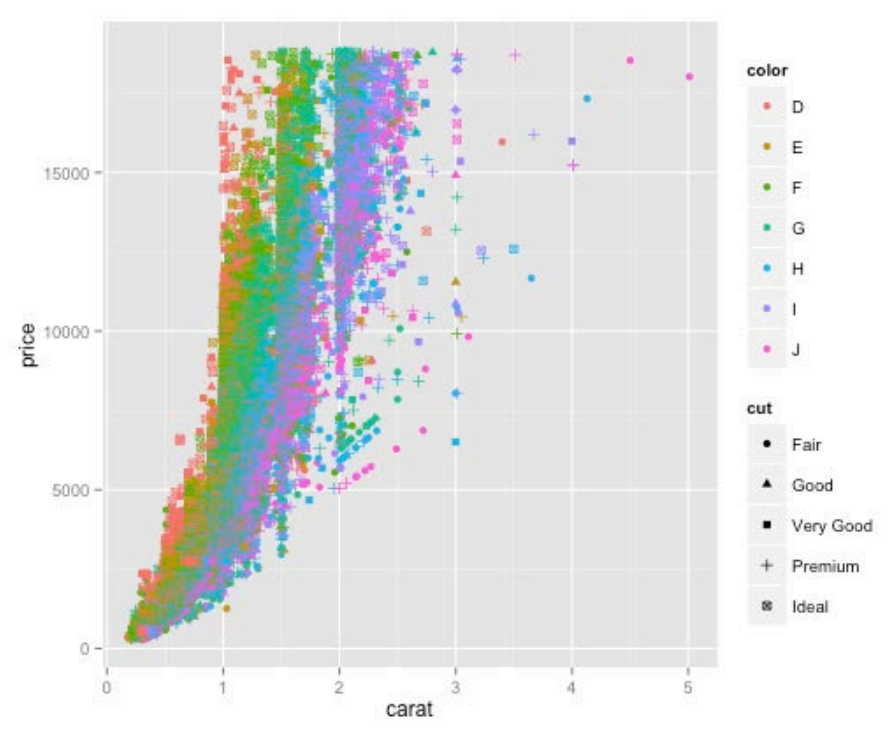




\section{qplot()}

$$
\begin{aligned}
& \text { qplot (carat, price, } \\
& \text { data = diamonds, } \\
& \text { geom =c("smooth")) }
\end{aligned}
$$

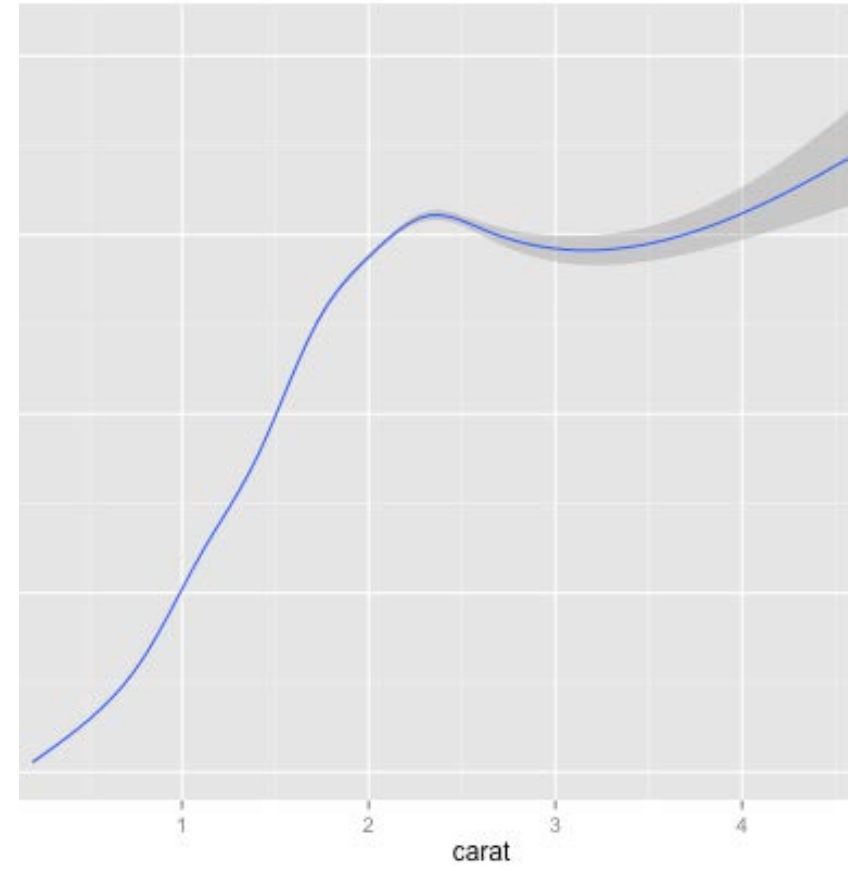




\section{qplot()}

qplot (carat, price,

data = diamonds,

geom $=$

c("point", "smooth"))

qplot (carat, price, data $=$ diamonds $)+$ geom_smooth()

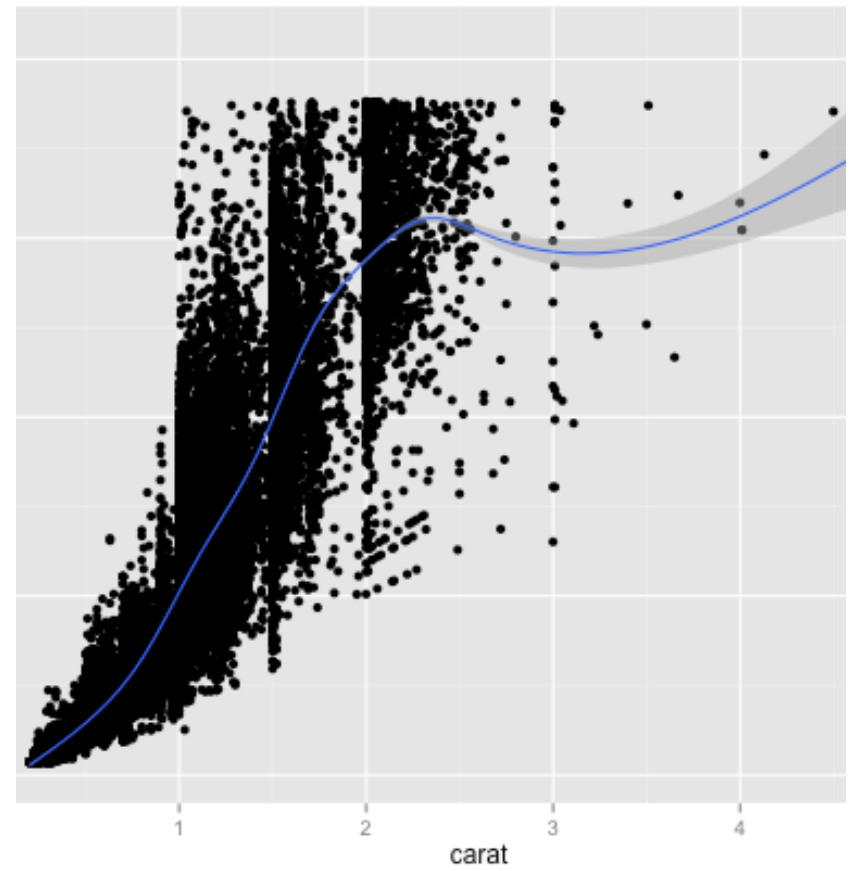




\section{Basic ggplot2}

Any layer will have the following:

- Data

- Aesthetic mappings e.g. diamond cut-> shape

- Geometrical objects. Points, bars, polygons, etc.

- Scales

- Coordinate system

- Faceting system 


\section{The ggplot2 syntax}

We'll start by assigning a ggplot to a variable

$$
\begin{aligned}
& \mathrm{p}<-\operatorname{ggplot}(\text { data }=\text { diamonds, aes }(\mathrm{x}=\text { carat })) \\
& \mathrm{p}<-\operatorname{ggplot}(\text { diamonds, aes }(\text { carat }))
\end{aligned}
$$




\section{The ggplot2 syntax}

$p+$

geom_histogram(

fill = "steelblue",

binwidth $=2$ ) 


\section{The ggplot2 syntax}

\begin{tabular}{|l|l|l|}
\hline geom & default stat & Aesthetics (required in bold) \\
\hline blank & identity & no parameters \\
\hline text & & $\begin{array}{l}\mathbf{x}, \mathbf{y}, \text { label, } \\
\text { size, color, alpha, hjust, vjust, } \\
\text { parse }\end{array}$ \\
\hline & identity & $\begin{array}{l}\mathbf{x}, \mathbf{y}, \\
\text { size, shape, color, fill, alpha, } \\
\text { na.rm }\end{array}$ \\
\hline point & identity & $\begin{array}{l}\mathbf{x}, \\
\mathbf{y}, \text { size, linetype, color, fill, alpha }\end{array}$ \\
\hline
\end{tabular}




\section{Scales}

p <- ggplot(diamonds, aes(carat, price,

$$
\begin{aligned}
& \text { color }=\text { cut })) \\
+ & \text { geom_point }()
\end{aligned}
$$

p

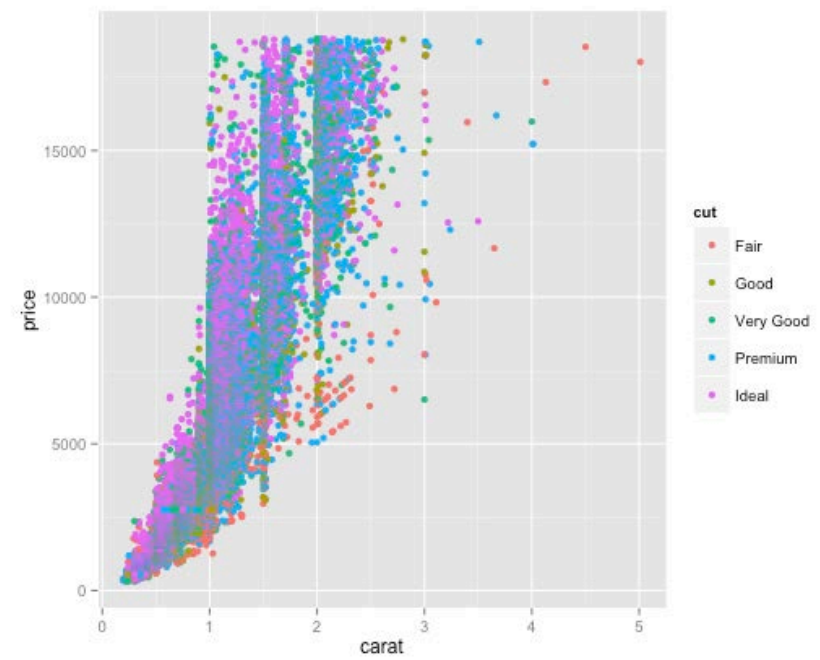




\section{Scales}

$$
\text { p + scale_y_log10() }
$$

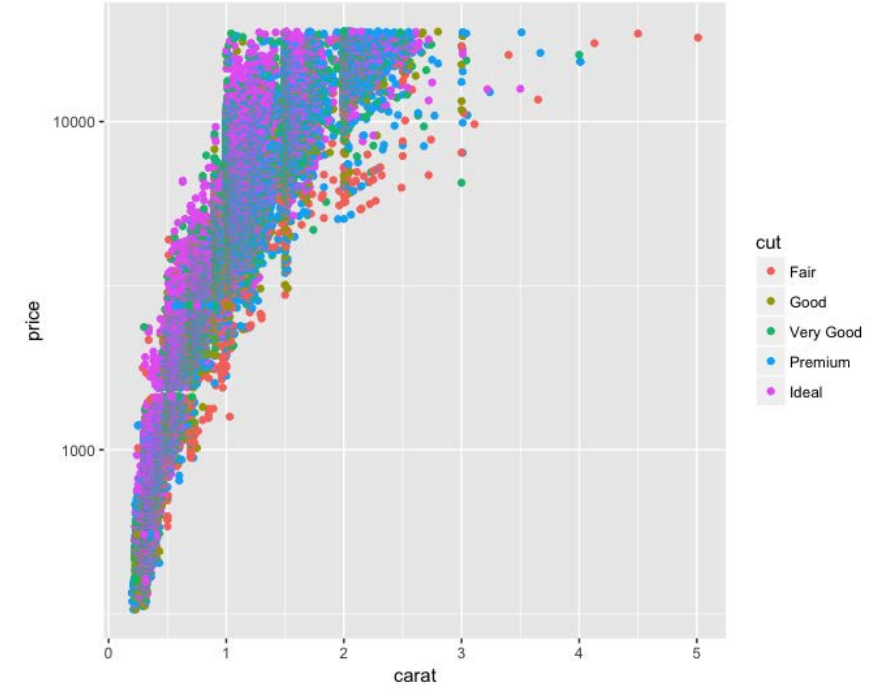




\section{Scales}

$$
p+\text { scale_x_log10( ) + scale_y_log10( ) }
$$

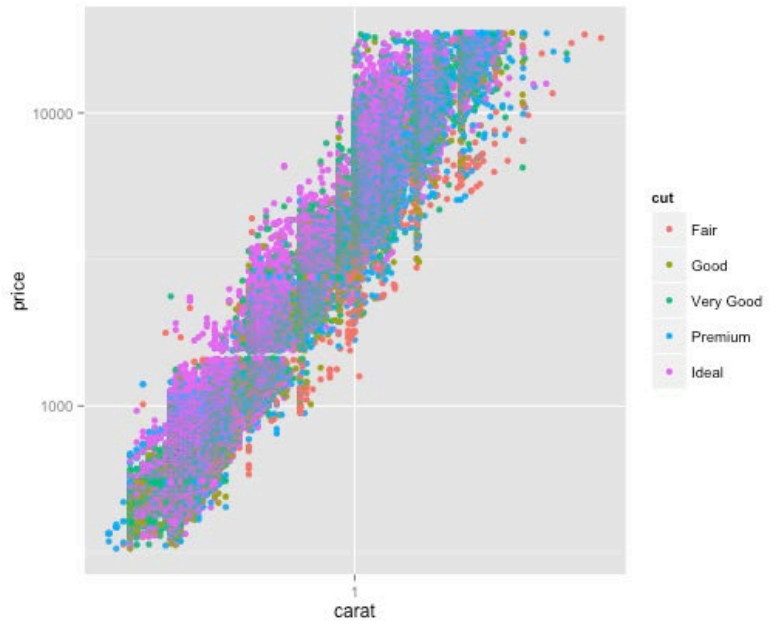




\section{Scales}

The color space in ggplot is hcl. In this space we have the following

Hue: an angle between 0 and 360 for color

Chroma: the "purity" of the color. At 0 you have grey. The maximum depends on luminance

Luminance: brightness. Black at 0 and white at 1 .

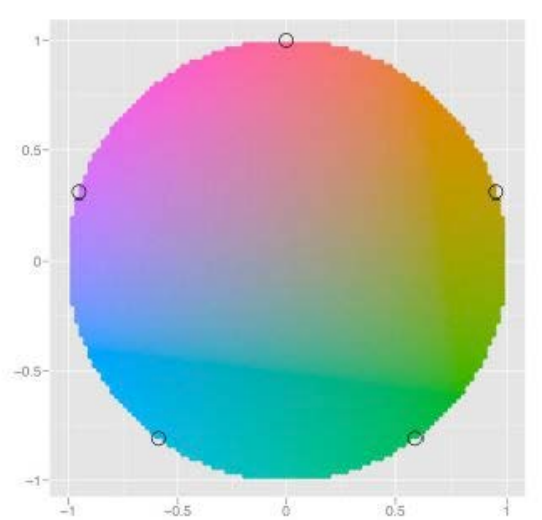




\section{Scales}

$$
p+\operatorname{scale} \text { _color_discrete }(h=c(0,360))
$$

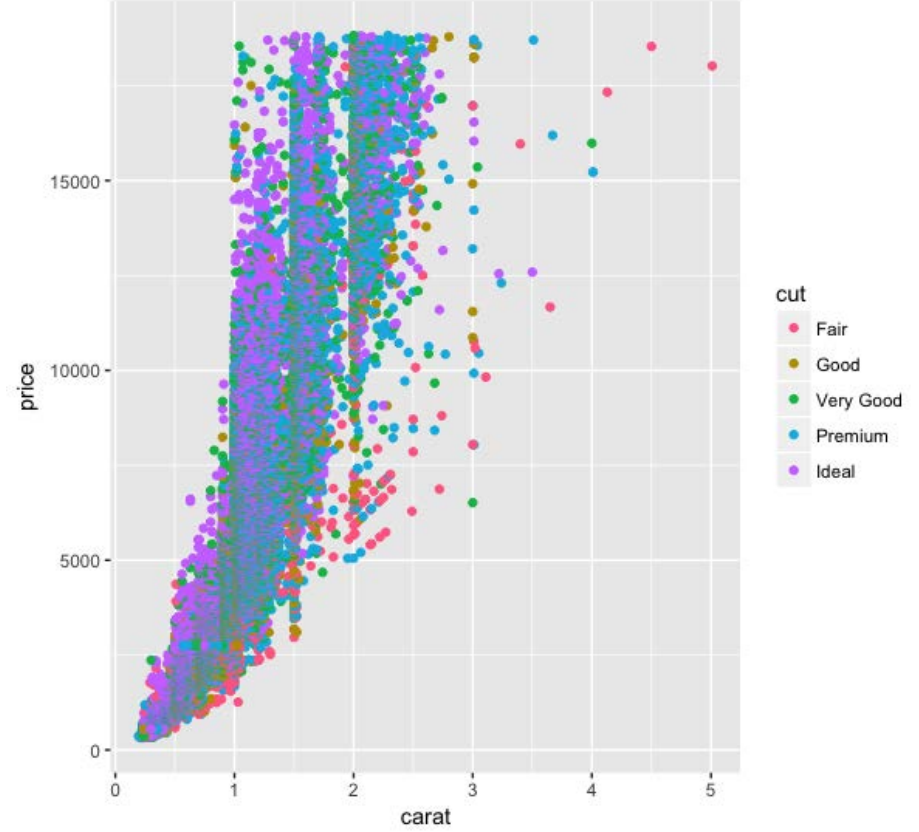




\section{Scales}

$$
p+\text { scale_color_discrete }(h=c(\theta, 180))
$$

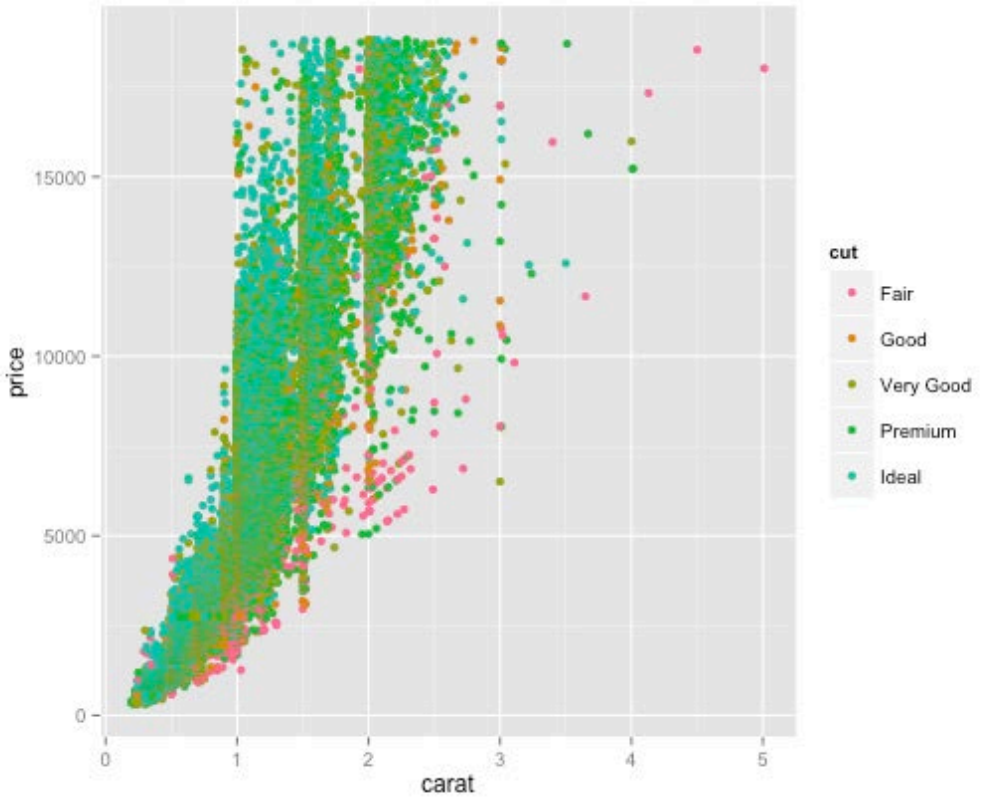




\section{Scales}

$$
p+\operatorname{scale} \text { ccolor_discrete }(h=c(0,30))
$$

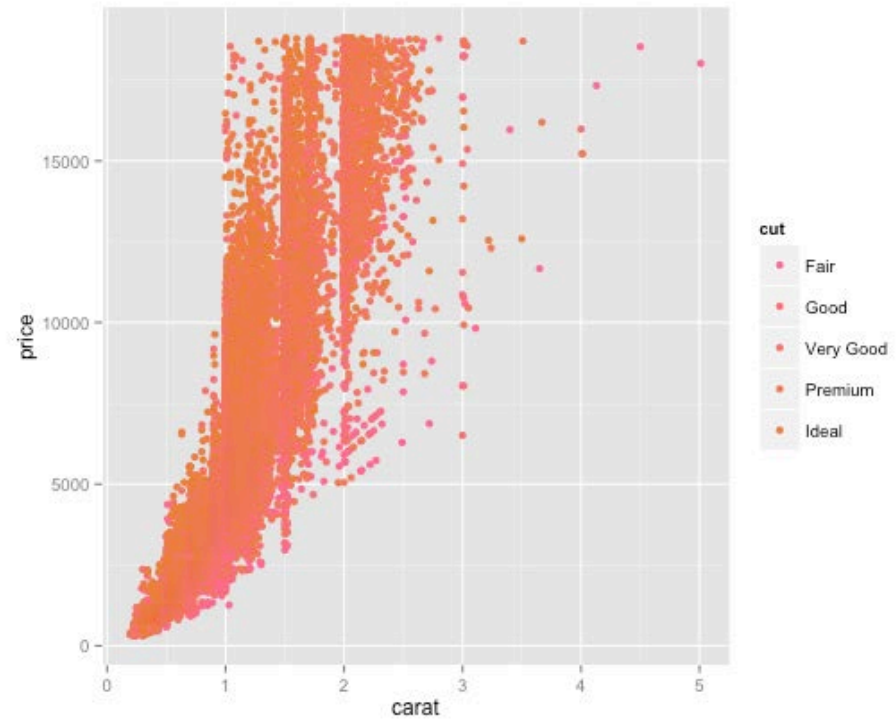




\section{Scales}

$$
\begin{aligned}
& p+\text { scale_color_discrete }(h=c(0,180), \\
& c=100,1=20)
\end{aligned}
$$

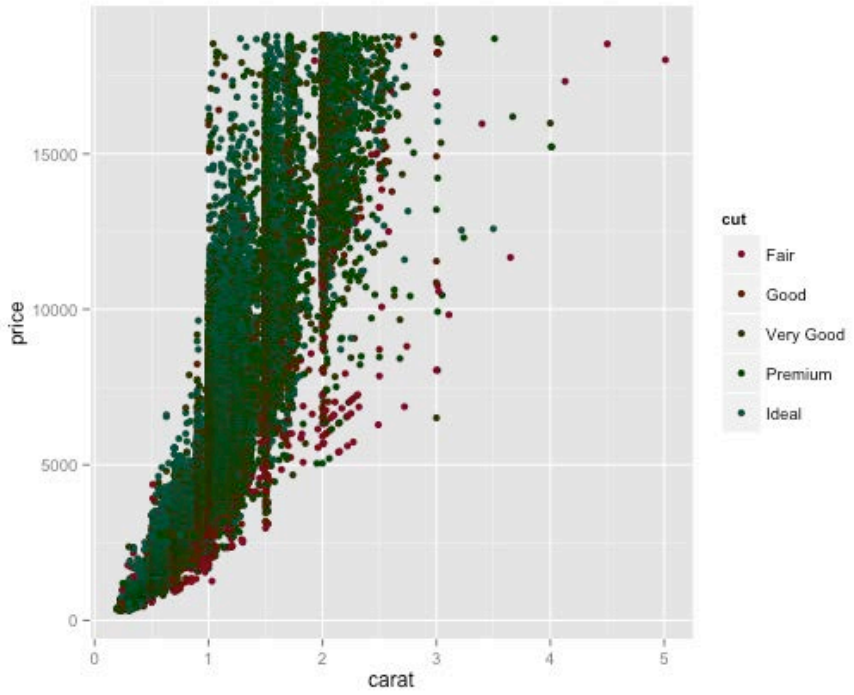




\section{Scales}

$$
\begin{aligned}
& p+\text { scale_color_discrete }(h=c(0,180), \\
& c=100, \bar{l}=100)
\end{aligned}
$$

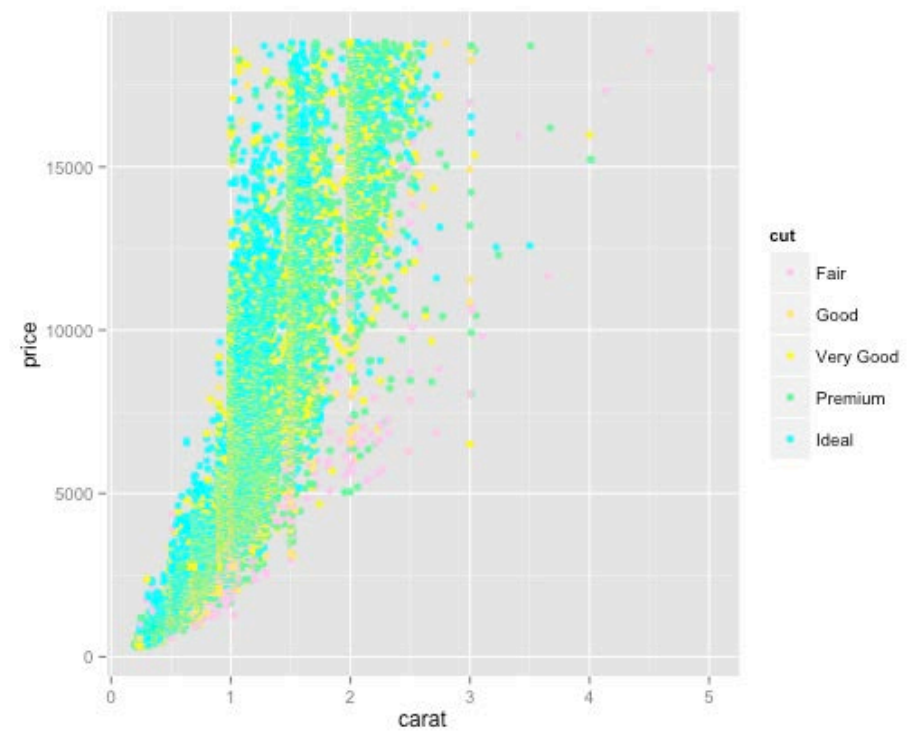




\section{Scales}

Cynthia Brewer's color palettes are available.

$p+$ scale_color_brewer(type = "div", palette $=4$ )

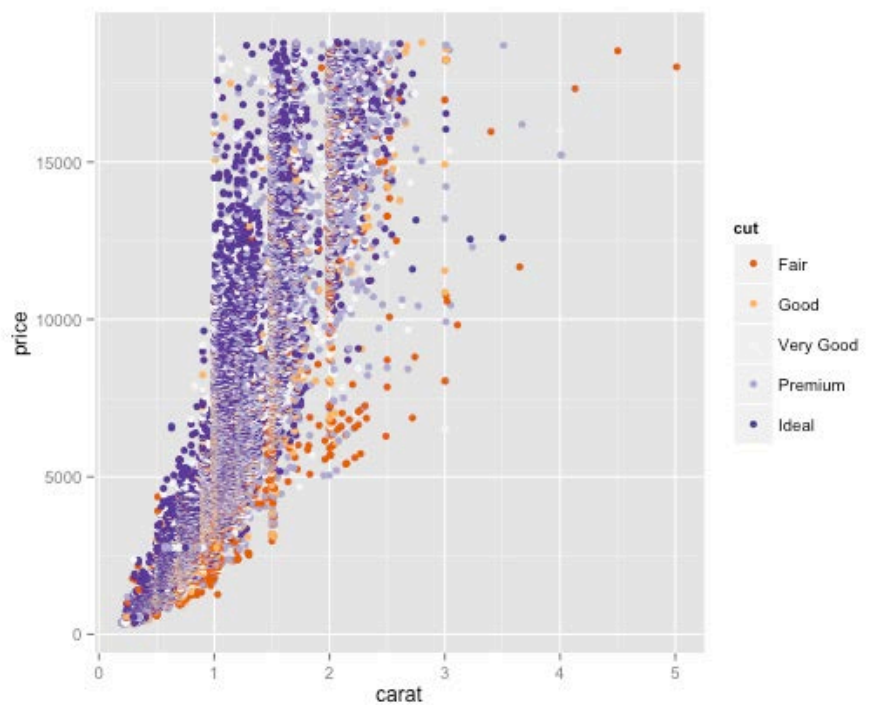




\section{Scales}

p + scale_color_brewer(type = "seq", palette $=4$ )

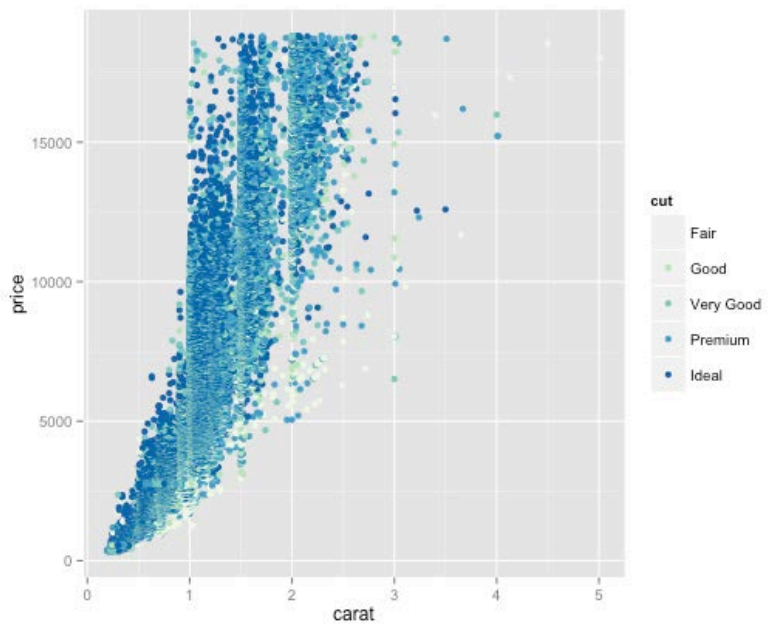




\section{Scales}

What about color for continuous values?

q <- ggplot(diamonds, aes(carat, price,

$$
\begin{aligned}
& \text { color }=\text { depth }) \text { ) } \\
& + \text { geom_point }()
\end{aligned}
$$

q

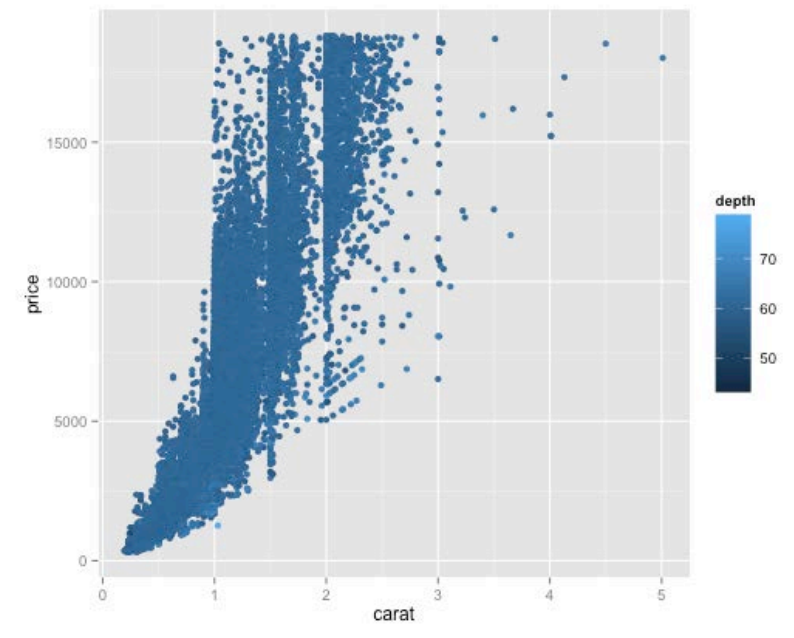




\section{Scales}

q + scale_color_continuous(low = "black", high = "pink"))

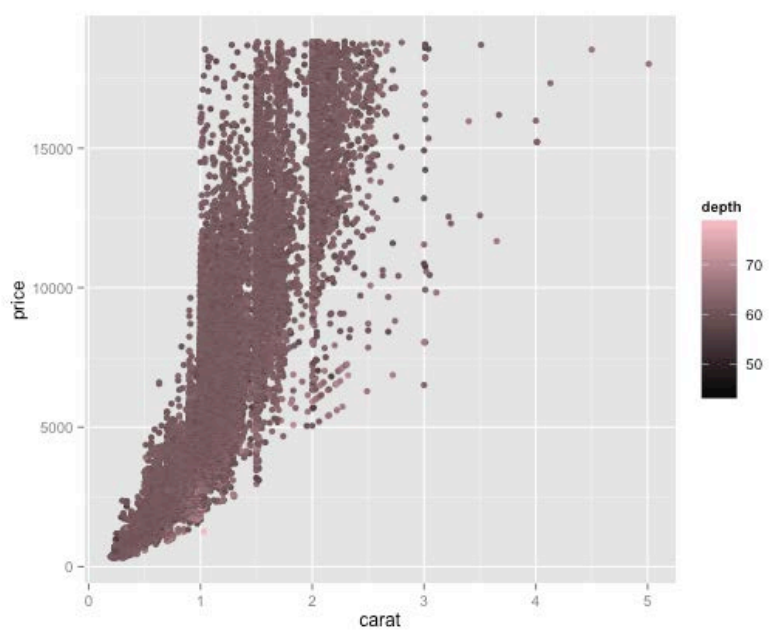




\section{Scales}

$$
\begin{aligned}
& q \text { + scale_color_gradientn(colors = c("red", } \\
& \text { "green", "steel blue")) }
\end{aligned}
$$

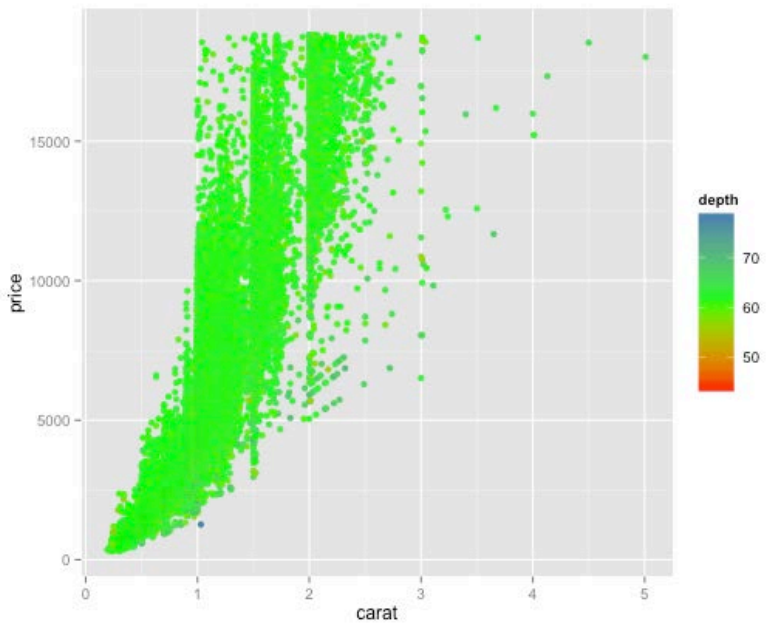




\section{Scales}

$q+$ scale_color_gradientn $($ colors $=$ terrain.colors (10))

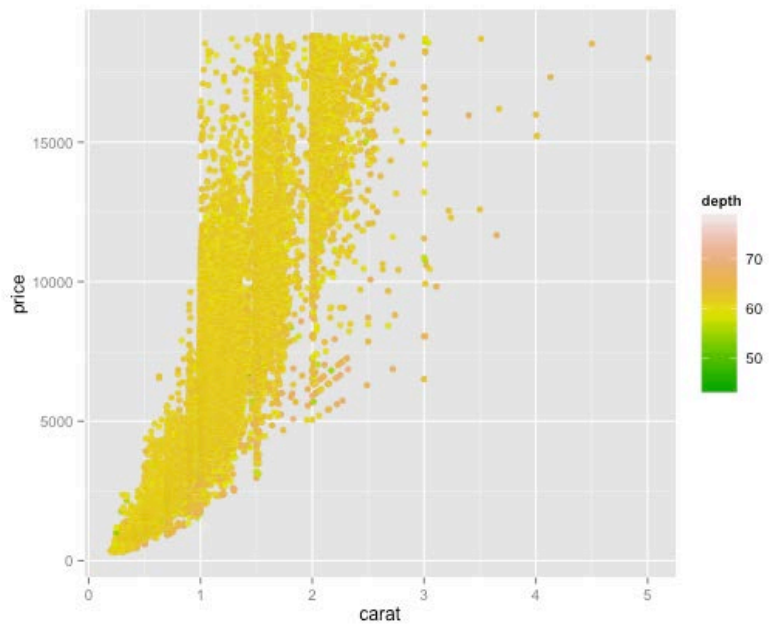




\section{Scales}

Try not to be too cute with the colors.

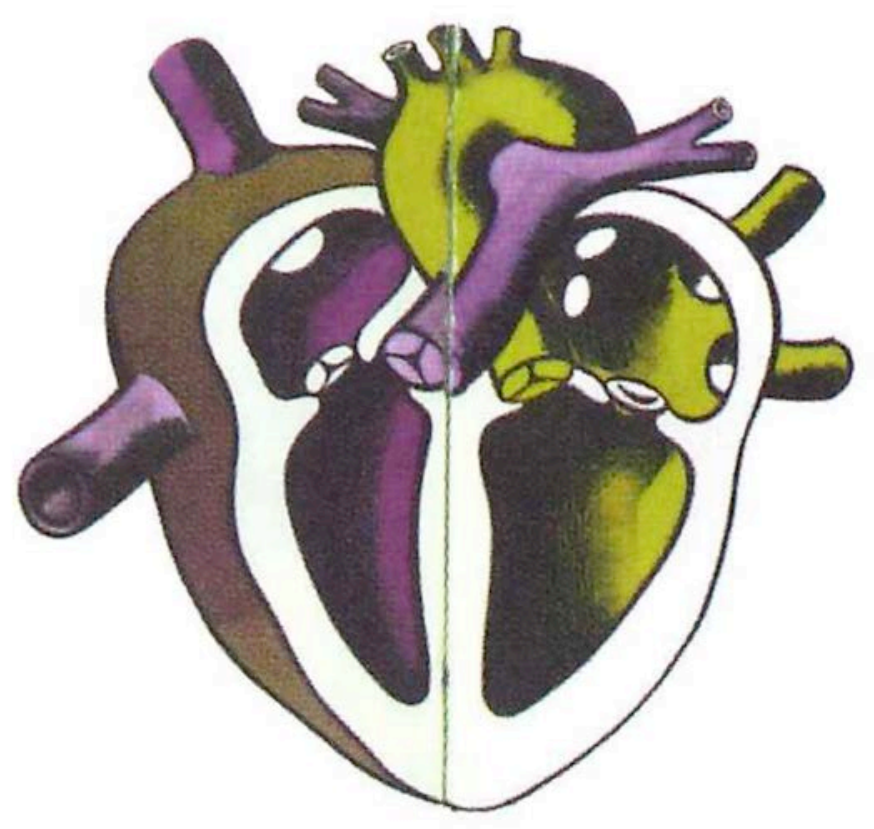




\section{Overplotting}

How to deal with overplotting?

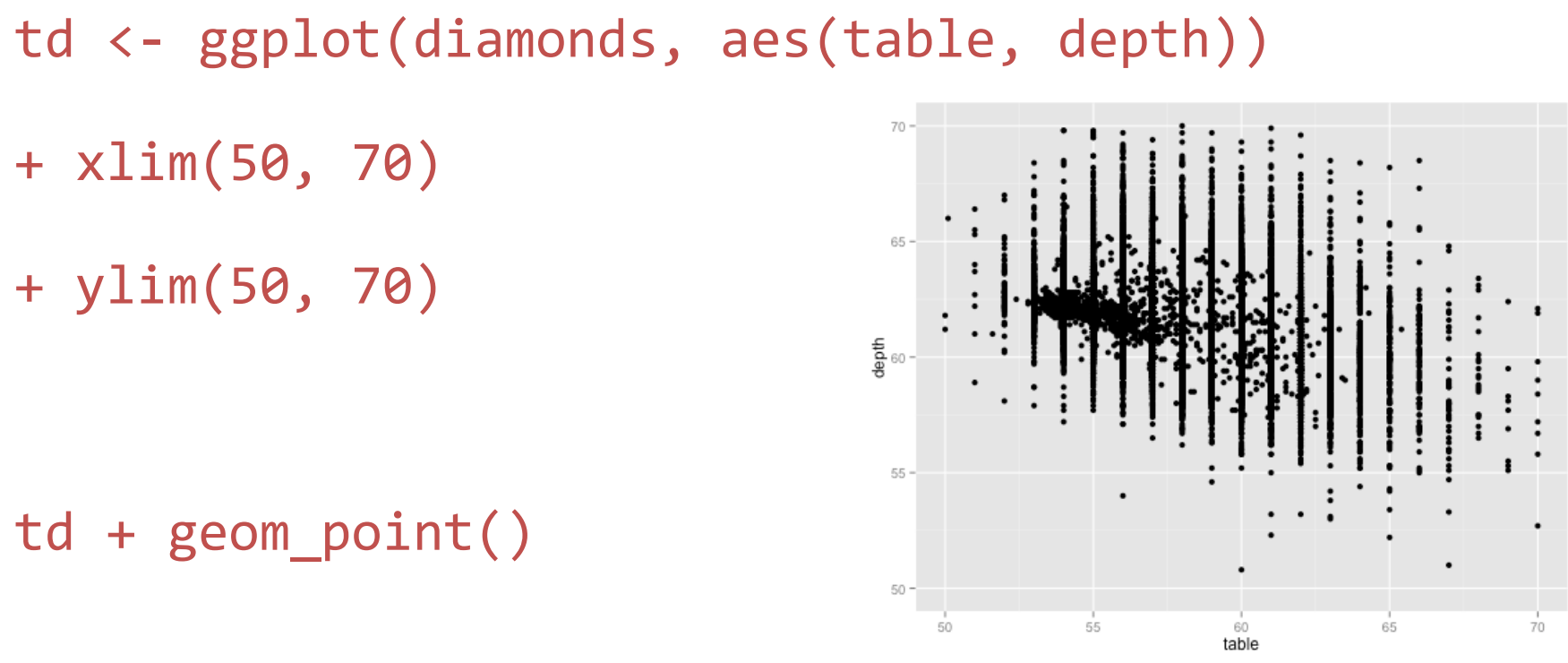




\section{Overplotting}

td + geom_jitter(position =

position_jitter $($ width $=.1)$ )

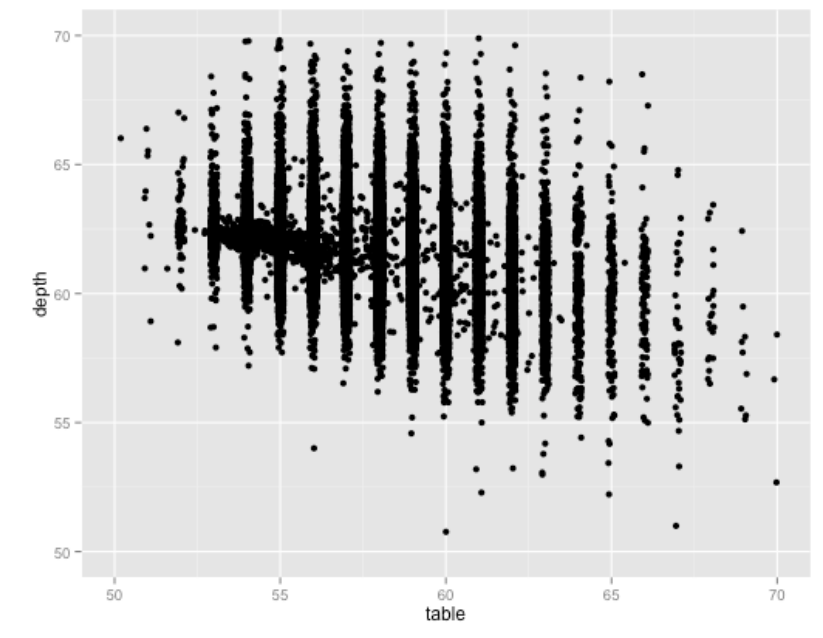




\section{Overplotting}

td + geom_jitter $($ position $=$ position_jitter (width $=.5$ ) ;

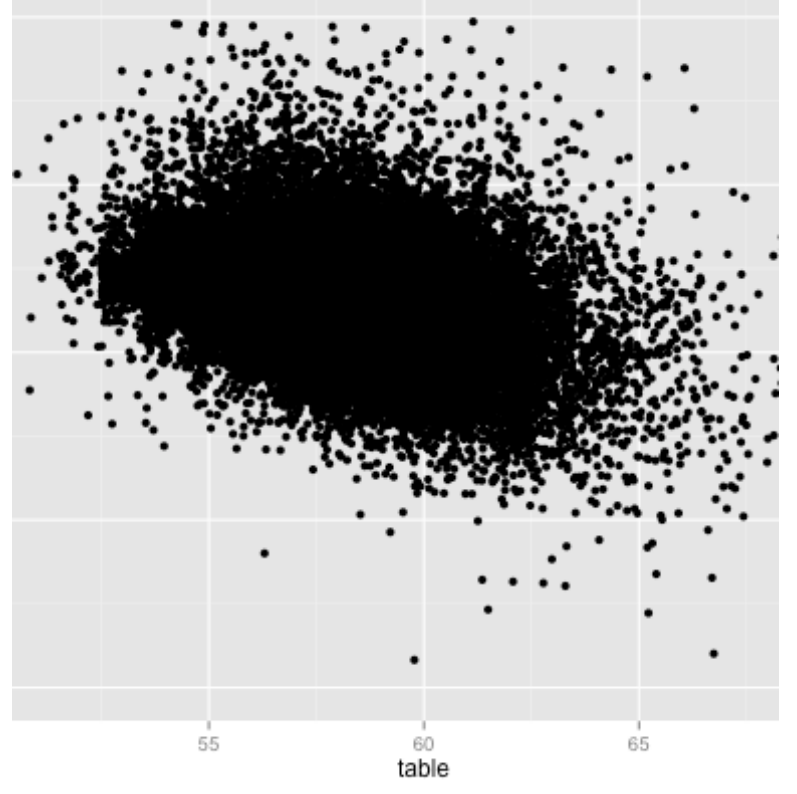




\section{Overplotting}

td + geom_jitter (position = position_jitter (

width $=.1$,

alpha $=.1$ )

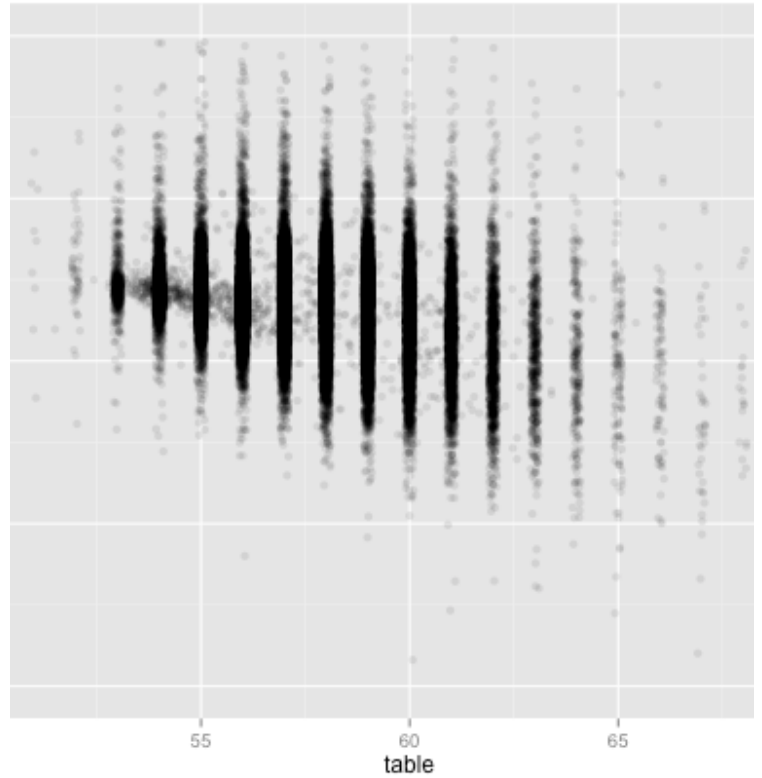




\section{Overplotting}

td + stat_bin2d()

\#This is the same as

\#td + geom_bin2d()

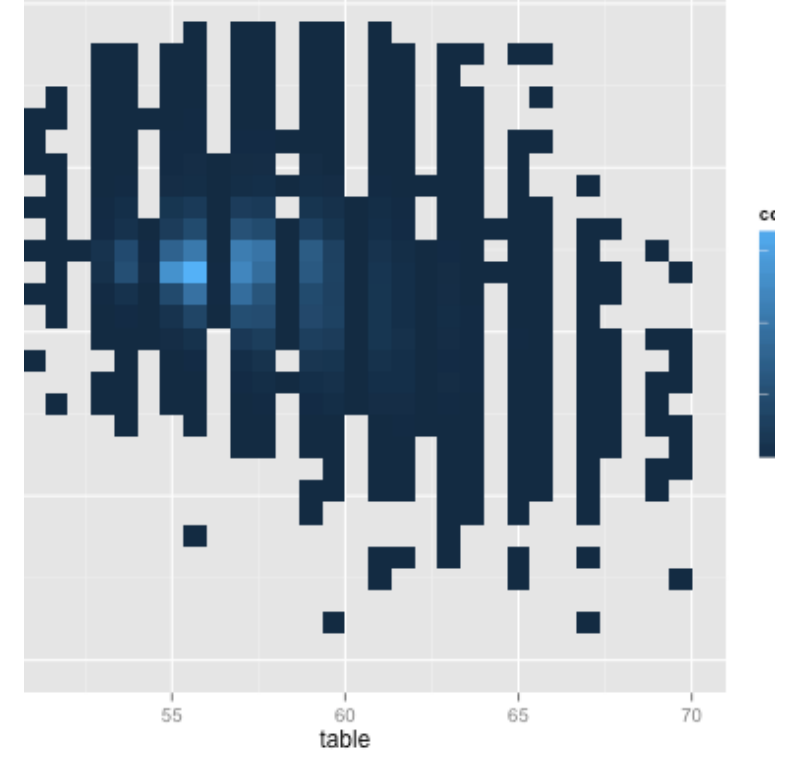



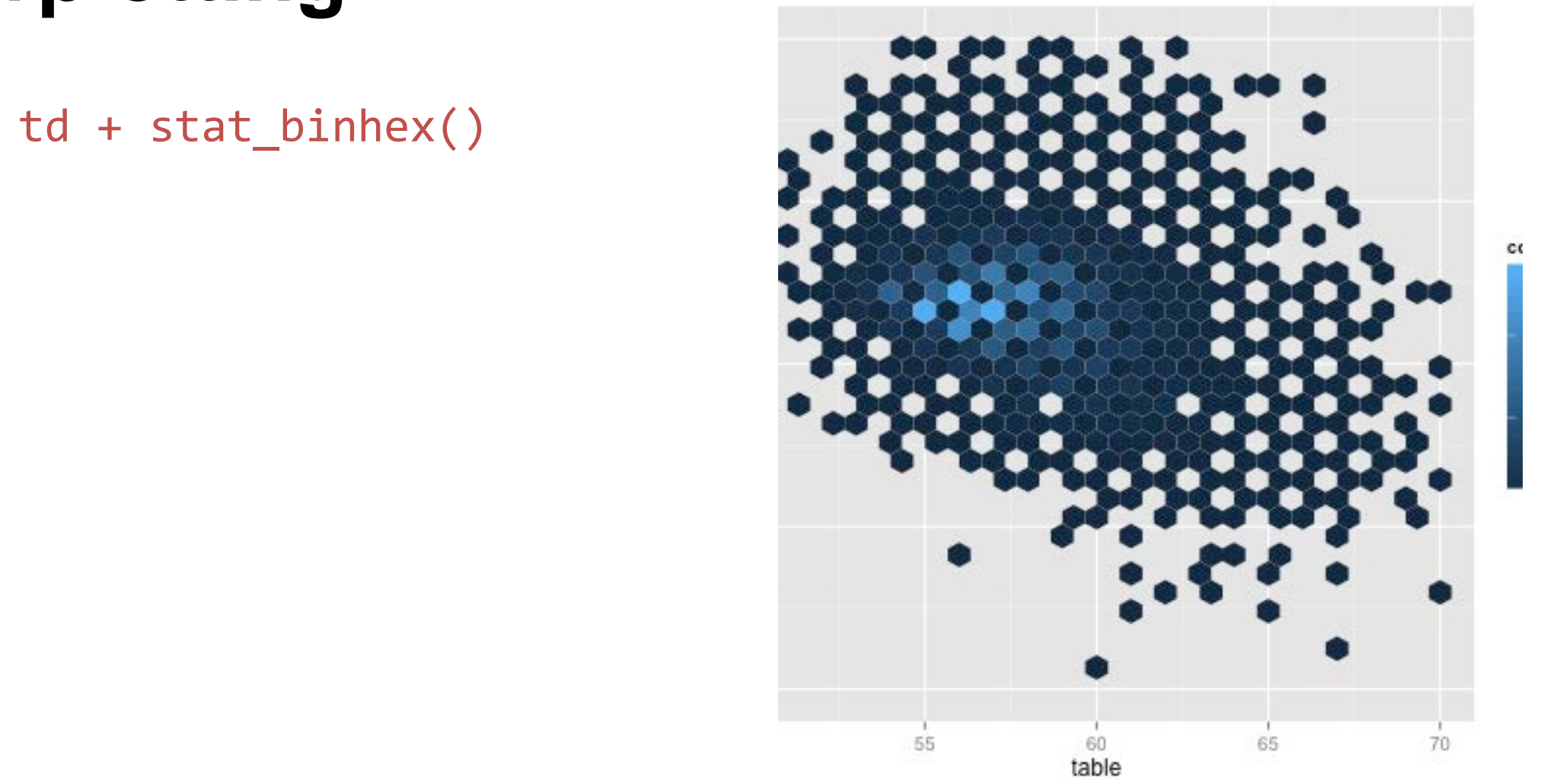


\section{Overplotting/Faceting}

qplot (carat, price, data = diamonds,

$$
\text { color }=\text { color })
$$




\section{Overplotting/Faceting}

qplot (carat, price,

data = diamonds,

color $=$ color $)+$

facet_grid(color . )

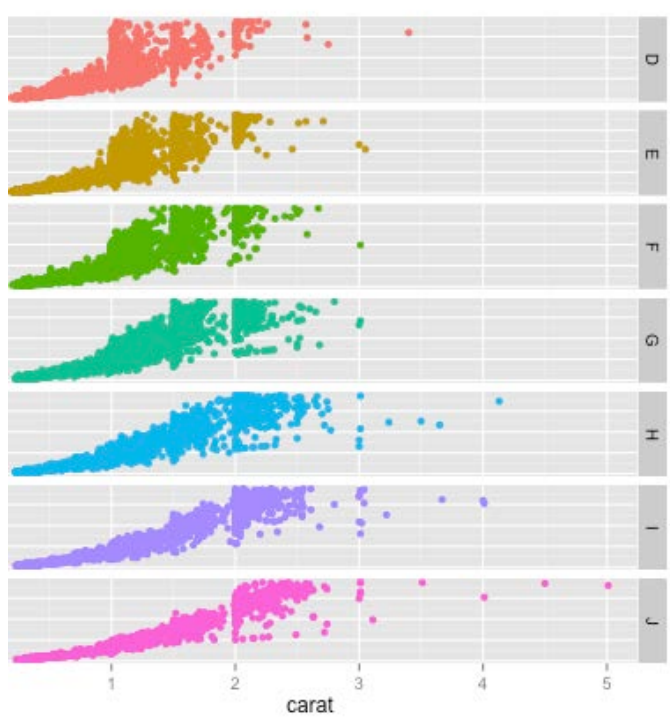




\section{Overplotting/Faceting}

qplot(carat, price,

$$
\begin{aligned}
& \text { data = diamonds, } \\
& \text { color = color })+ \\
& \text { facet_grid }(. \sim \text { cut })
\end{aligned}
$$

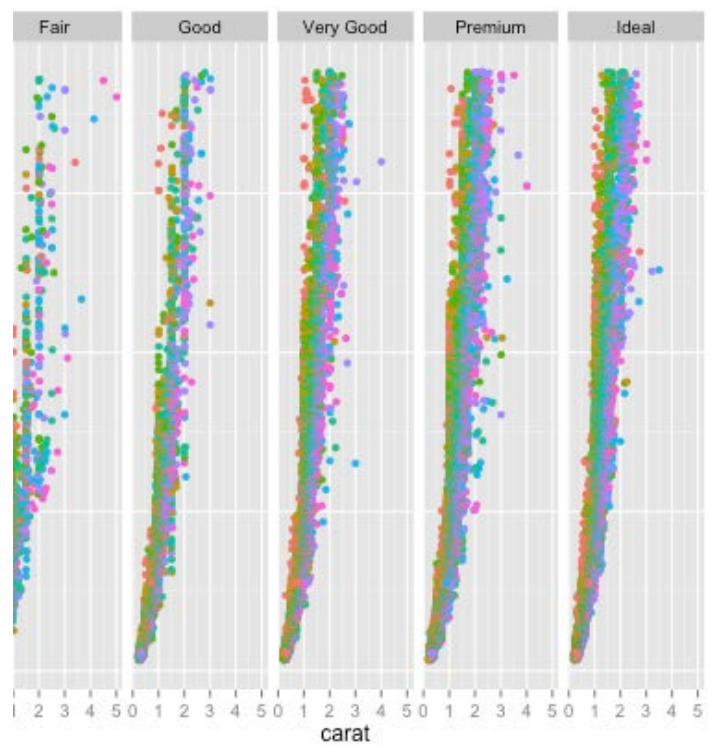




\section{Overplotting/Faceting}

qplot(carat, price,

data = diamonds,

color $=$ color $)+$ facet_grid $($ color $\sim$ cut $)$

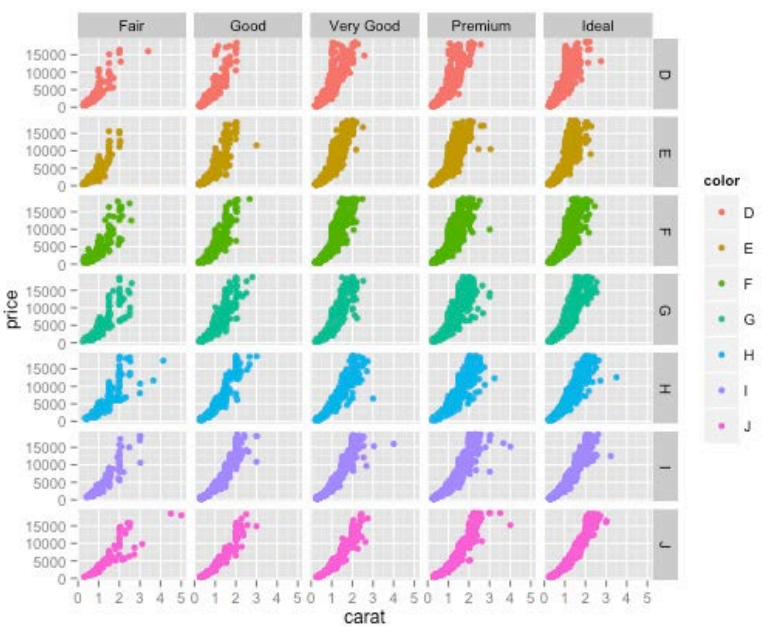




\section{Revisiting Minard}

While Tufte says Minard's Napoleon graph tracks six dimensions, Wilkinson points out there is a grouping variable so we have seven values with aesthetic mappings.

troops <- read.table("troops.txt", header=TRUE)

cities <- read.table("cities.txt", header=TRUE) 


\section{Revisiting Minard}

\section{head(troops)}

long lat survivors direction group

$\begin{array}{llllll}1 & 24.0 & 54.9 & 340000 & \text { A } & 1\end{array}$

$\begin{array}{lllll}2 & 24.5 & 55.0 & 340000 & \text { A }\end{array}$

$\begin{array}{lllll}3 & 25.5 & 54.5 & 340000 & \text { A }\end{array}$

$\begin{array}{lllll}4 & 26.0 & 54.7 & 320000 & \text { A }\end{array}$

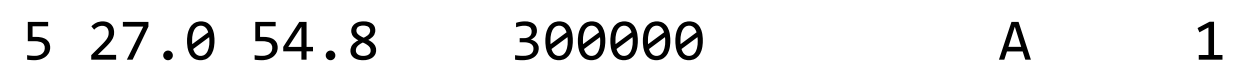

$\begin{array}{lllll}6 & 28.0 & 54.9 & 280000 & \text { A }\end{array}$ 


\section{Revisiting Minard}

head(cities)

long lat city

$124.055 .0 \quad$ Kowno

$\begin{array}{llll}2 & 25.3 & 54.7 \quad \text { Wilna }\end{array}$

326.454 .4 Smorgoni

426.854 .3 Moiodexno

527.755 .2 Gloubokoe

$627.653 .9 \quad$ Minsk 


\section{Revisiting Minard}

\#Adapted from code by Hadely Wickham

ggplot(cities, aes $(x=$ long, $y=$ lat $))+$

geom_path(aes(size = survivors, color = direction, group $=$ group $)$, data $=$ troops $)+$

geom_point ( ) +

geom_text (aes(label $=$ city $),$ hjust $=0$, vjust $=1$, size=4) +

scale_size $($ range $=c(1,10))+$

scale_colour_manual (values $=c($ "grey", "red")) +

scale_x_continuous (limits $=c(24,39)$ ) 


\section{Revisiting Minard}

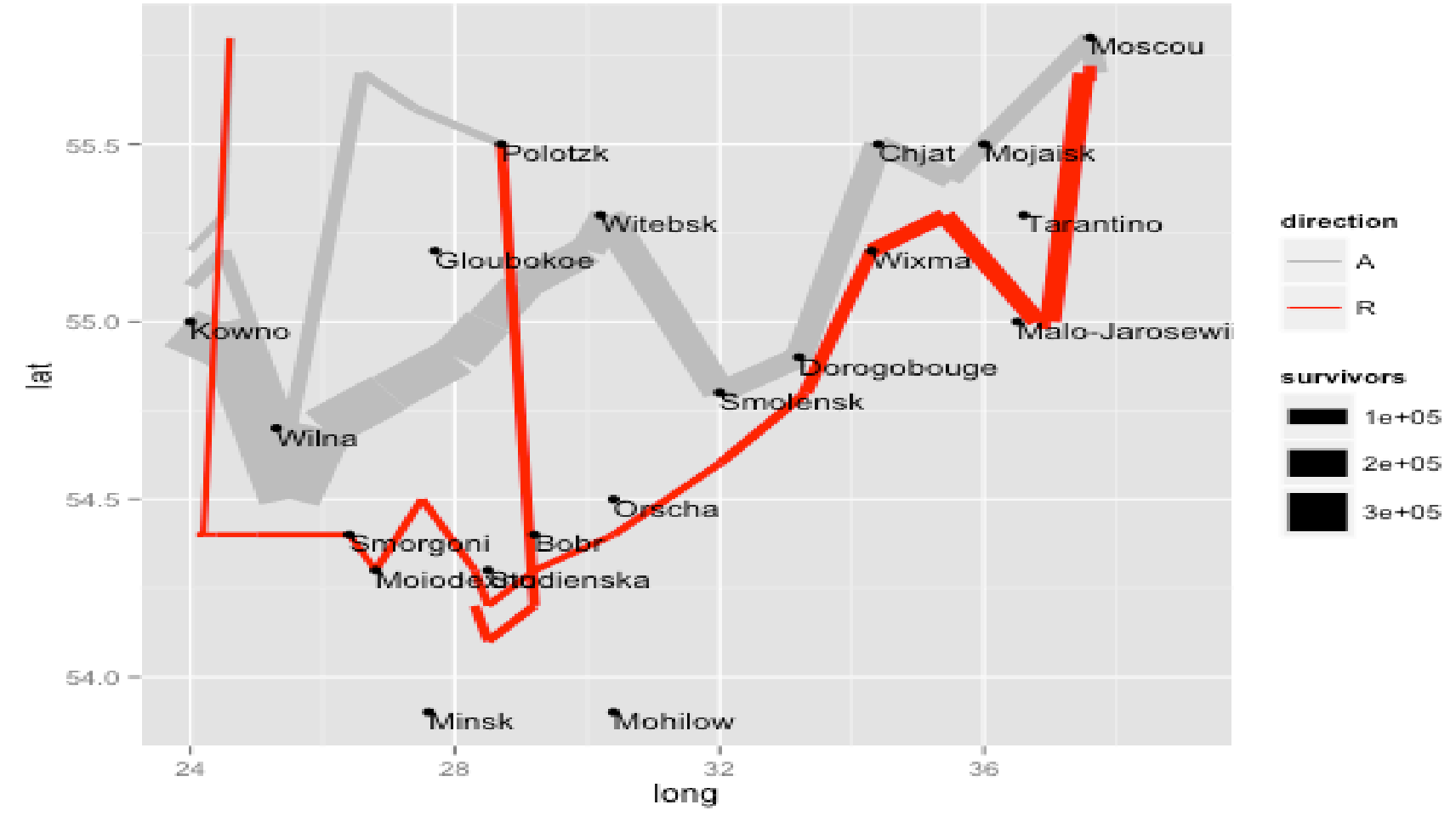




\section{Revisiting Minard}

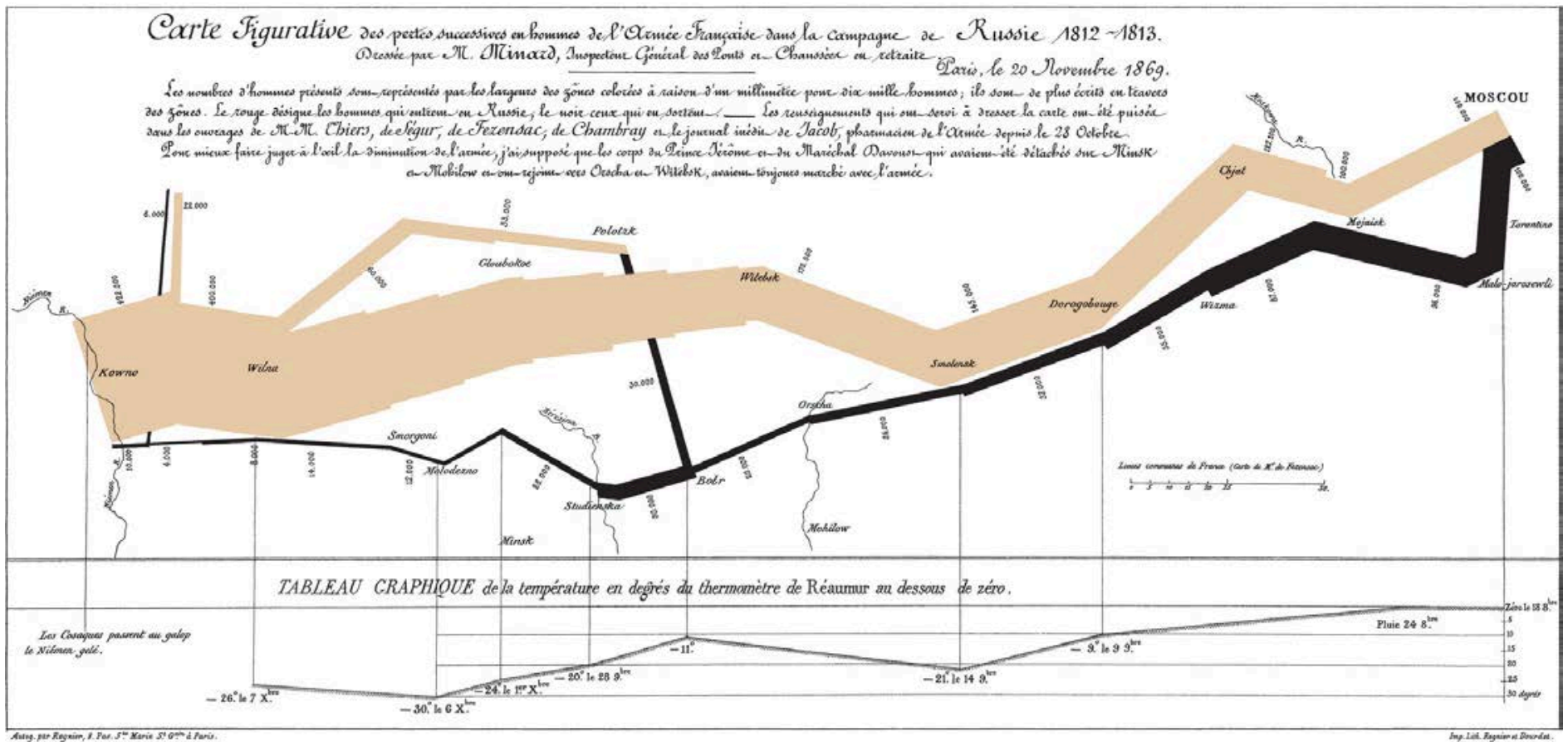


David Reagan, Advanced Visualization Lab

Web tools for

Information Visualization 


\section{Why build something for the web?}

- Accessibility

- Let users interact with results

- Let users do their own exploration or analysis

"Overview first, zoom and filter, then details-on-demand" 


\section{Can't I do that with Shiny?}

- If you use $\mathrm{R}$, then you probably can

- If you use Python, try Dash or Bokeh 


\section{When to use Shiny}

- Comfortable in the R ecosystem, not interested in learning new tools

- Need the power of the server, not worried about having to run Shiny server

- Don't need anything too fancy 


\section{Prepare data for the web}

- Export flat files in JSON or CSV

- Find import library for your data type (SheetJS, ...)

- Query database 


\section{Mr. Data Converter}

I will convert your Excel data into one of several web-friendly formats, including HTML, JSON and XML.

Fork me on github.

SETTINGS

Delimiter: ○ Auto • Comma • Tab

Decimal Sign: ○ Dot $\bullet$ Comma

v First row is the header

Transform: $\bullet$ downcase $\bullet$ upcase $\bullet$ none

a Include white space in output

Indent with: $\bullet$ tabs $\circ$ spaces
Input CSV or tab-delimited data. Using Excel? Simply copy and paste. No data on hand? Use sample

$\begin{array}{llll}\text { NAME } & \text { VALUE } & \text { COLOR } & \text { DATE } \\ \text { Alan } & 12 & \text { blue } & \text { Sep. 25, 2009 } \\ \text { Shan } & 13 & \text { "green } & \text { blue" Sep. 27, } 2009 \\ \text { John } & 45 & \text { orange } & \text { Sep. 29, 2009 } \\ \text { Minna } & 27 & \text { teal } & \text { Sep. 30, } 2009\end{array}$

Output as JSON - Properties

[ \{ "NAME" : "Alan", "VALUE" : 12, "COLOR" : "blue", "DATE": "Sep. 25, 2009"\},

\{"NAME" : "Shan", "VALUE": 13, "COLOR": "green\tblue", "DATE" : "Sep. 27, 2009"\},

\{ "NAME" : "John", "VALUE" : 45, "COLOR": "orange", "DATE" : "Sep. 29, 2009"\},

\{"NAME" : "Minna", "VALUE" : 27, "COLOR" : "teal", "DATE": "Sep.30, 2009"\} ]

\section{】J indiana university}




\section{D3: Data-Driven Documents}

- JavaScript library for manipulating web documents using data

- Uses open standards: HTML, CSS, SVG

- Typically renders SVG, but can render to HTML canvas for performance

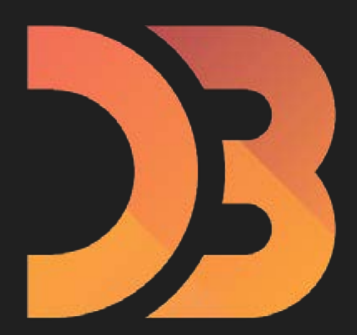




\section{An interactive example}

- Bind data to standard web elements

- Update data to update existing elements, add new ones, delete old ones

- Maintain object constancy with smooth transitions

- https://bl.ocks.org/mbostock/raw/3808234/

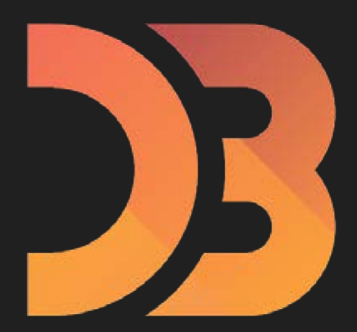




\section{Example: Define data}

var alphabet = "abcdefghijklmnopqrstuvwxyz".split("");

IT indiana unIVERSITY 


\section{Example: Update data on timer}

// Grab a random sample of letters from the alphabet, in alphabetical order. d3. interval (function() \{

update (d3.shuffle (alphabet)

.slice (0, Math.floor (Math.random () * 26)) . sort ());

\}, 1500);

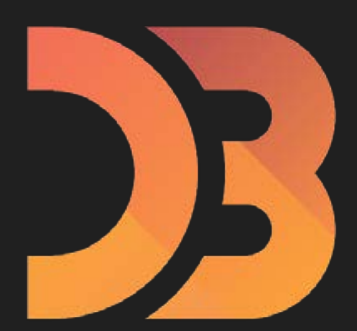

$\Psi$ 


\section{Example: Create transition}

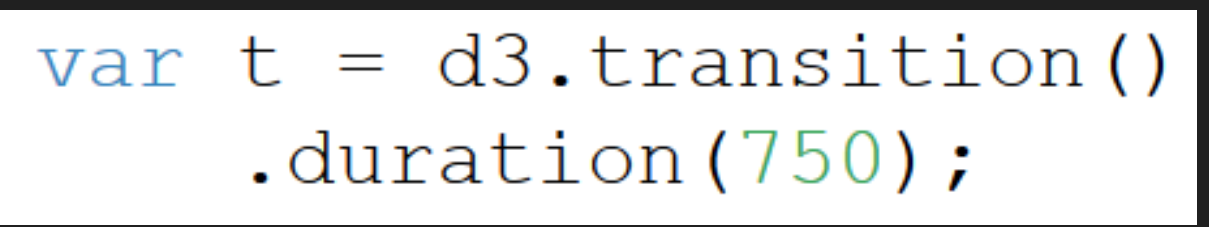




\section{Example: Update selection with new data}

// JOIN new data with old elements.

var text $=$ g.selectAll ("text")

- data(data, function(d) \{return d; \});

TI INDIANA unIVERSITY 


\section{Example: Remove old elements}

// EXIT old elements not present in new data. text.exit()

-attr("class", "exit")

-transition ( $t$ )

.attr("Y", 60)

. style("fill-opacity", 1e-6)

.remove () ; 


\section{Example: Update old elements}

// UPDATE old elements present in new data.

text.attr("class", "update")

. attr ("Y", 0$)$

.style("fill-opacity", 1)

.transition(t)

. attr("x", function $(d, i)\{\operatorname{return} i * 32 ;\})$; 


\section{Example: Create new elements}

// ENTER new elements present in new data.

text.enter () . append ("text")

. attr("class", "enter")

. $\operatorname{attr}(" \mathrm{dy} ", " .35 \mathrm{em} ")$

. $\operatorname{attr}(" Y ",-60)$

- attr("x", function $(d, i) \quad\{\operatorname{return} i * 32 ;\})$

.style("fill-opacity", 1e-6)

.text (function (d) \{return d; \})

- transition ( $t)$

- attr ("y", 0$)$

.style("fill-opacity", 1); 


\section{A more InfoVis-y version of the same idea}

1. https://bost.ocks.org/mike/nations/

2. https://romsson.github.io/dragit/example/nations.html

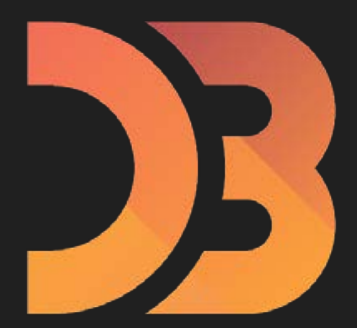




\section{But how do I make graphs?}

- Bar: append('rect')

- Line: append('path')

- Scatter: append('circle')

- Everything by hand! 


\section{Vega and Vega-Lite}

- "Vega is a visualization grammar"

- "Vega-Lite is a high-level grammar of interactive graphics"

- Both use a JSON specifications to map data to properties of graphical marks

- Vega-Lite can be compiled to Vega

- Both use D3 underneath 


\section{Vega-Lite: Scatter}
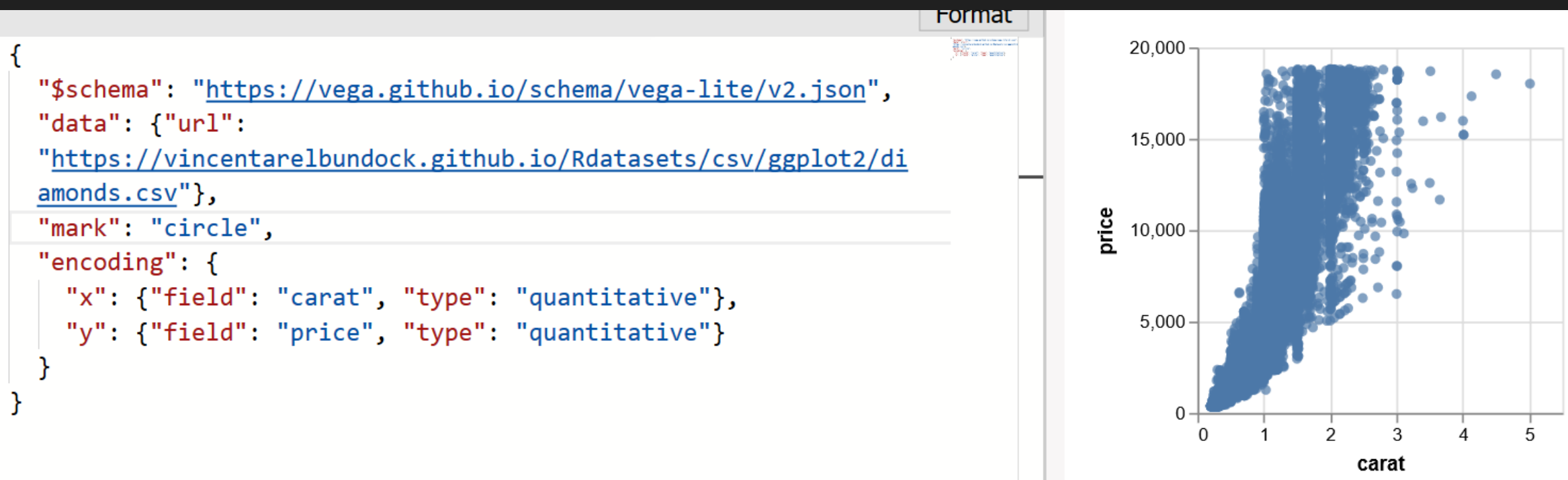


\section{Vega-Lite: Scatter}

\{

"\$schema":

"https://vega.github.io/schema/vega-lite/v2.json", "description": "A scatterplot showing horsepower and miles per gallons.",

"data": \{ "url":

"https://vincentarelbundock.github.io/Rdatasets/csv/ ggplot2/diamonds.csv"\}, "mark": "point",

"encoding": \{

"x": \{"field": "carat", "type": "quantitative"\}, "y": \{"field": "price", "type": "quantitative"\}, "color": \{"field": "color", "type": "nominal"\}, \}

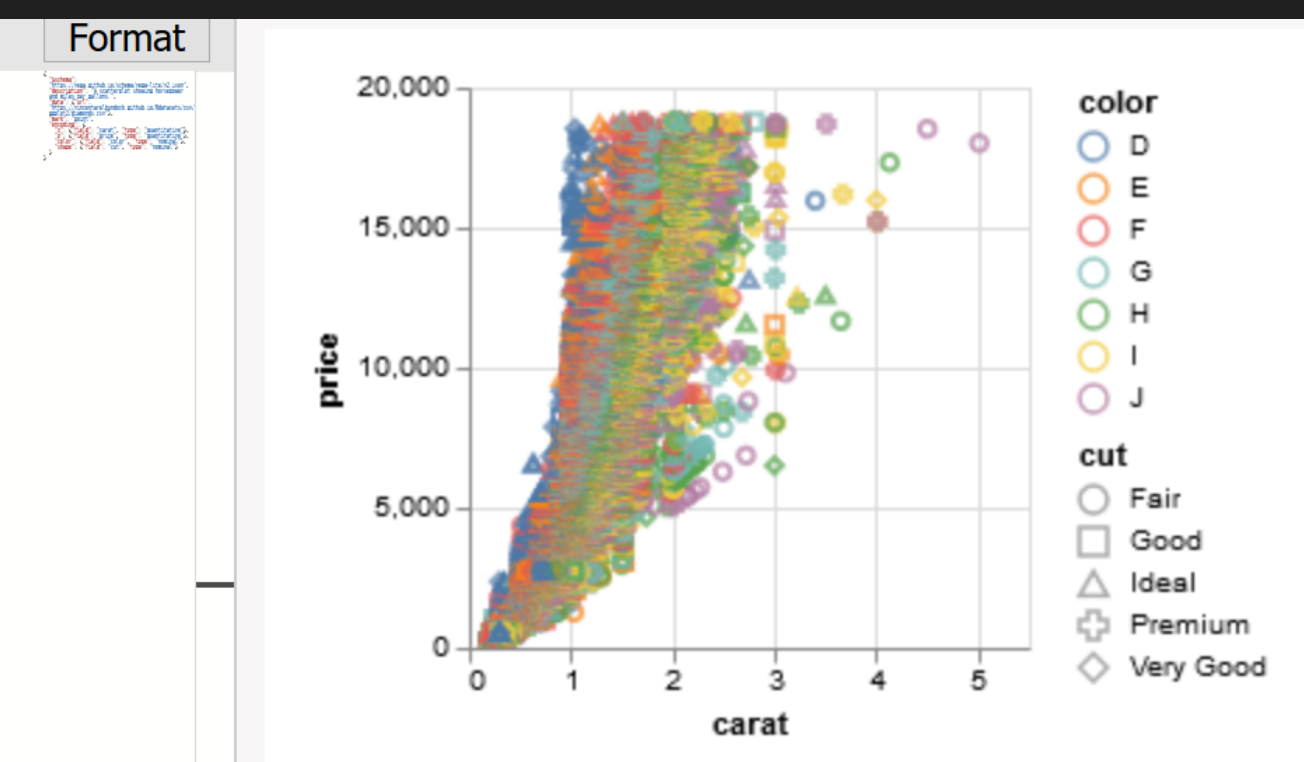




\section{Vega-Lite: Histogram}

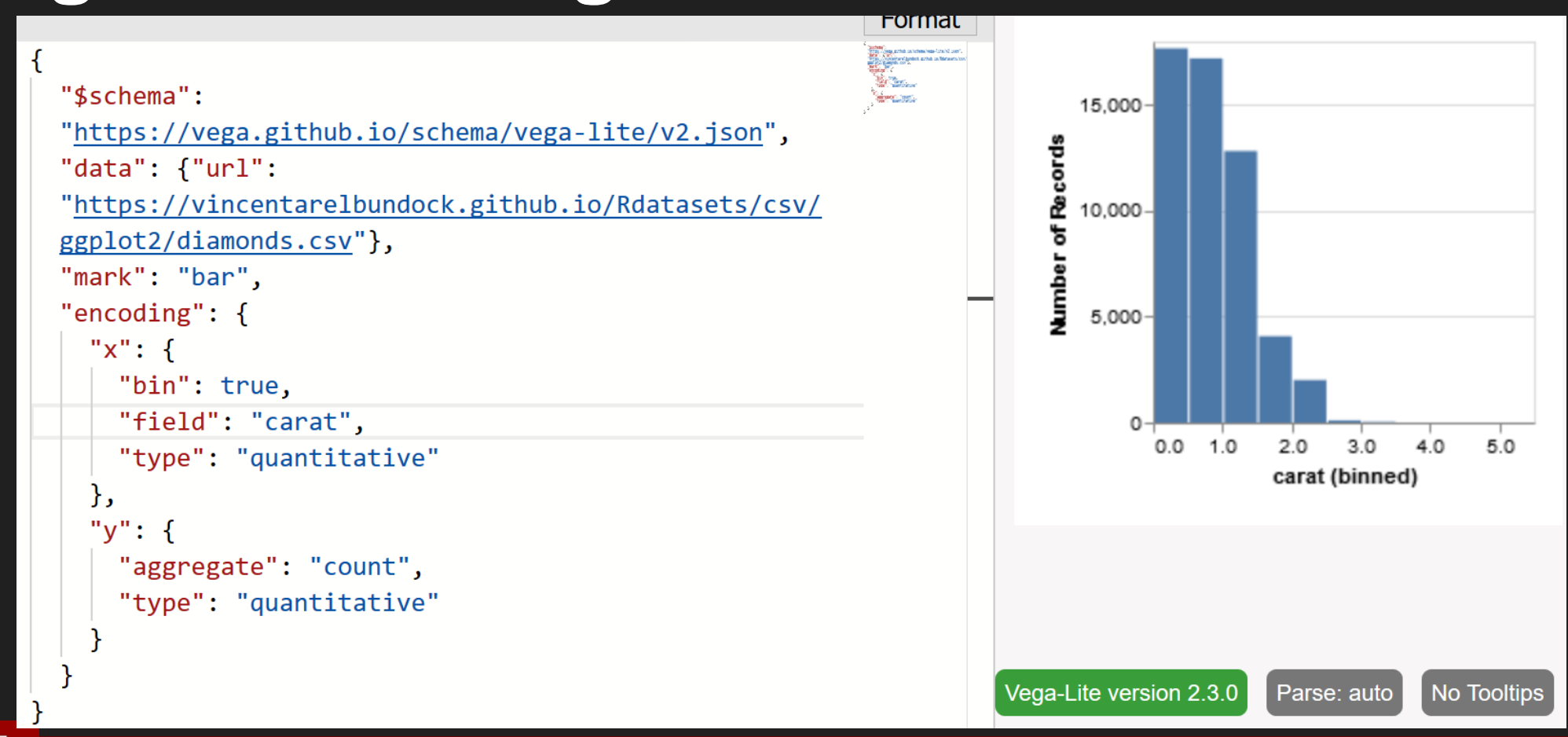




\section{Vega-Lite: Scatter with filter}

\{

"\$schema": "https://vega.github.io/schema/vega-lite/v2.json", "data": \{"url":

"https://vincentarelbundock.github.io/Rdatasets/csv/ggplot2/d iamonds.csv"\},

"mark": "circle",

"transform": [

\{"filter": $\{$ "field": "table", "range": [50, 70]\}\},

\{"filter": $\{$ "field": "depth", "range": [50, 70]\}\} ],

"encoding": \{

"x": \{"field": "table", "type": "quantitative", "scale": \{"domain": [50, 70]\}\},

"y": \{"field": "depth", "type": "quantitative", "scale": $\{$ "domain": [50,70]\}\}

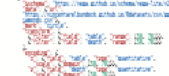

Bets

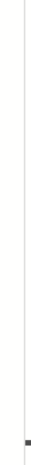

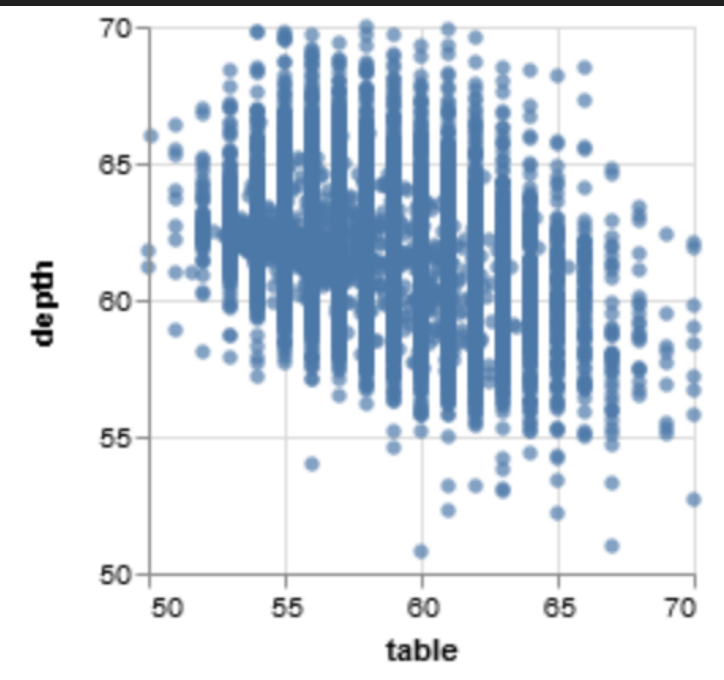
\}

$\Psi$ 


\section{Vega-Lite: Scatter with lower opacity}

$\left\{\begin{array}{l}\{ \\ \} \\ \}\end{array}\right.$

"\$schema": "https://vega.github.io/schema/vega-lite/v2.json", "data": \{"url":

"https://vincentarelbundock.github.io/Rdatasets/csv/ggplot2/d iamonds.csv" \},

"mark": \{"type": "circle", "opacity": 0.1$\}$,

"transform": [

\{"filter": \{"field": "table", "range": [50,70]\}\},

\{"filter": \{"field": "depth", "range": [50, 70]\}\}

],

"encoding": \{

"x": \{"field": "table", "type": "quantitative",

"scale": \{"domain": [50,70]\}\},

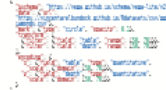

"y": \{"field": "depth", "type": "quantitative",

"scale": \{"domain": [50,70]\}\} \}

\}

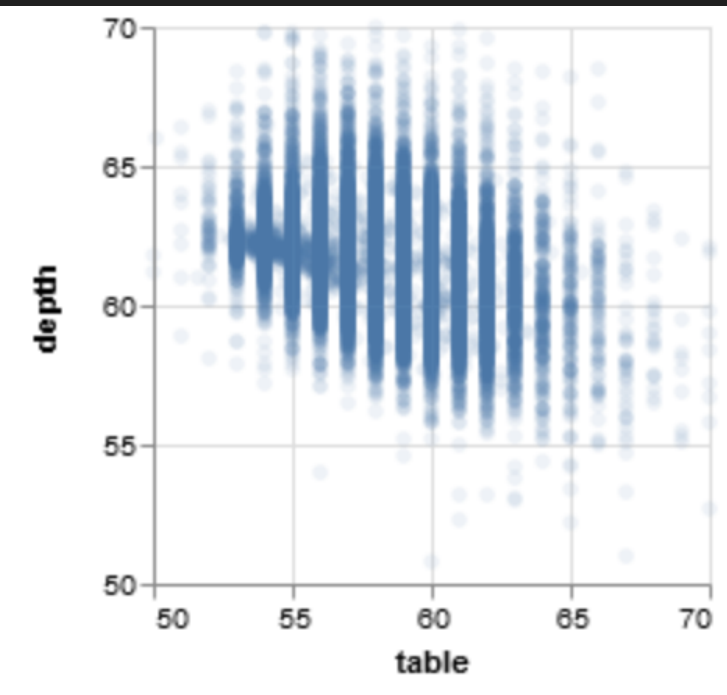

$\Psi$ 


\section{Vega-Lite: Scatter with bins}

"mark": \{"type": "rect"\},

"transform": [

\{"filter": \{"field": "table", "range": [50, 70]\}\},

\{"filter": \{"field": "depth", "range": [50, 70]\}\} ],

"encoding": \{

"x": \{"field": "table", "type": "quantitative", "scale": \{"domain": [50, 70]\}, "bin":

$\{$ "maxbins": 40\}\},

"y": \{"field": "depth", "type": "quantitative", "scale": \{"domain": [50, 70]\}, "bin":

$\{$ "maxbins": 40\}\},

"color": \{"aggregate": "count",

"type": "quantitative"\}

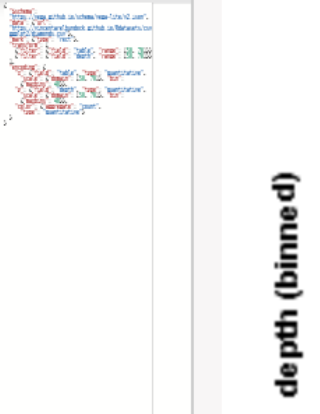

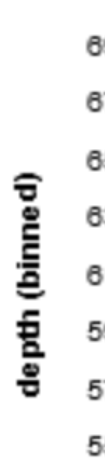

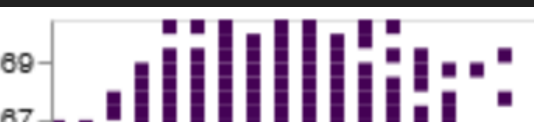
in (I) . 政 )

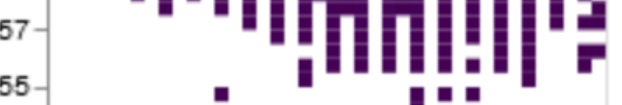

53 $51+\frac{}{50}$
Number of Record

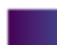

2,427 


\section{Vega-Lite: Scatter with facets}

"width": 30, "height": 20,

"mark": \{"type": "circle", "size": 1\}, "encoding": \{

"x": \{"field": "carat", "type":

"quantitative" $\}$,

"y": \{"field": "price", "type":

"quantitative" $\}$,

"color": \{"field": "color", "type": "nominal"\}

,

"column": \{"field": "color", "type":

"nominal" $\}$,

"row": \{"field": "cut", "type": "nominal"\}
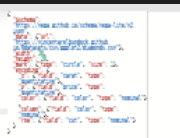

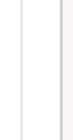

$-$

莫竞

ن

:

$>$

\section{color}

$=$ : 20,000

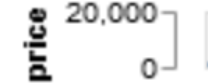

i。 20,000

는

递 $\begin{array}{r}20,000 \\ \text { ㄴ. }\end{array}$

蒫 20,000$]$

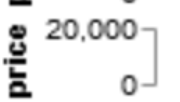

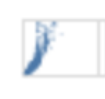

5

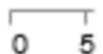

$\longdiv { 5 }$

carat carat carat

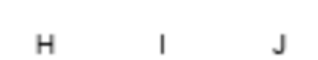

